\title{
Lattice initial segments of the hyperdegrees
}

\author{
Richard A. Shore* \\ Department of Mathematics \\ Cornell University \\ Ithaca NY 14853
}

\author{
Bjørn Kjos-Hanssen ${ }^{\dagger}$ \\ Department of Mathematics \\ University of Hawai'i at Mānoa \\ Honolulu HI 96822
}

October 18, 2018

\begin{abstract}
We affirm a conjecture of Sacks [1972] by showing that every countable distributive lattice is isomorphic to an initial segment of the hyperdegrees, $\mathcal{D}_{h}$. In fact, we prove that every sublattice of any hyperarithmetic lattice (and so, in particular, every countable locally finite lattice) is isomorphic to an initial segment of $\mathcal{D}_{h}$. Corollaries include the decidability of the two quantifier theory of $\mathcal{D}_{h}$ and the undecidability of its three quantifier theory. The key tool in the proof is a new lattice representation theorem that provides a notion of forcing for which we can prove a version of the fusion lemma in the hyperarithmetic setting and so the preservation of $\omega_{1}^{C K}$. Somewhat surprisingly, the set theoretic analog of this forcing does not preserve $\omega_{1}$. On the other hand, we construct countable lattices that are not isomorphic to an initial segment of $\mathcal{D}_{h}$.
\end{abstract}

*Partially supported by NSF Grant DMS-0554855.

${ }^{\dagger}$ Partially supported as co-PI by NSF grant DMS-0652669.

The authors also thank the referee for suggestions that improved the presentation in several ways. 


\section{Contents}

1 Introduction $\quad 2$

2 Usl representations and the notion of forcing $\quad 9$

3 The fusion lemma $\quad 15$

4 Initial segment verifications $\quad 17$

5 The lattice representation theorem $\quad 21$

6 Usls and other questions $\quad 27$

\section{Introduction}

Given a notion of relative computability or complexity $\leq_{r}$ on sets $A$ (of natural numbers) or functions $f$ (from $\omega$ to $\omega$ ) the corresponding degree structure $\mathcal{D}_{r}$ consists of the equivalence classes $\operatorname{deg}_{r}(A)\left(\operatorname{deg}_{r}(f)\right)$ of mutually computable sets (functions) with the ordering induced by the given reducibility $\leq_{r}$. These classes are called the $r$-degrees. The classic example is Turing computability, Turing reducibility $\leq_{T}$ and the Turing degrees but many others have been studied ranging from polynomial-time to constructibility. The first level of the scaffolding on which our understanding of such degree structures is built provides an analysis of the partial orders that can be embedded in them.

For the Turing degrees the story begins with Kleene and Post [1954] who proved that every countable partial order can be embedded in $\mathcal{D}_{T}$. They used finite approximation methods that today would be called Cohen forcing in arithmetic. Stronger results about embedding uncountable partial orders were proven, for example, by Sacks [1963] but the full question of whether every partial order of size $2^{\aleph_{0}}$ with $\{x \mid x \leq z\}$ countable for every $z$ can be embedded in $\mathcal{D}_{T}$ remains open. Nonetheless, the countable case is more than enough to decide all one quantifier sentences of $\left\langle\mathcal{D}_{T}, \leq_{T}\right\rangle$ as being true if and only if consistent with the axioms of partial orders.

The next level of analysis deals with extension of embedding questions such as density or minimality and, more generally, questions about when a given realization of a partial order in $\mathcal{D}$ can be extended to a specified larger partial order. In $\mathcal{D}_{T}$, the long journey along this road began with Spector's [1956] construction of a minimal degree. His method can be viewed as using full binary trees in place of finite characteristic functions for the approximations to the desired set. These methods were greatly extended by many researchers to embed more and more lattices as initial segments of $\mathcal{D}_{T}$. We mention a few of the key steps: Lachlan [1968] showed that every countable distributive lattice is isomorphic to an initial segment of $\mathcal{D}_{T}$ (and so that its theory is undecidable); 
Lerman [1971] did the same for all finite lattices; Lachlan and Lebeuf [1976] for countable uppersemilattices (usls) and Abraham and Shore [1986] for all usls of size $\aleph_{1}$. (Groszek and Slaman [1983] show that it is consistent with ZFC that there are lattices of size $\aleph_{2} \leq 2^{\aleph_{0}}$ with $\{x \mid x \leq z\}$ countable for every $z$ that can not be embedded in $\mathcal{D}_{T}$ as initial segments.) The embedding methods in the countable situations involve approximations by more and more complicated types of trees whose shape is tied to a series of representation theorems for (uppersemi)lattices. The uncountable ones need, in addition, some set theoretic techniques.

At this level of our scaffolding, Lerman's [1971] result on embedding finite lattices shows that the three quantifier theory of $\mathcal{D}_{T}$ is undecidable (Schmerl, see Lerman [1983]). Coupled with the methods of Kleene and Post [1954], it also suffices to establish the decidability of the two quantifier theory of this structure (Shore [1978] and Lerman [1983, VII.4]). The case of countable recursive lattices suffices (Miller, Nies and Shore [2004]) to show the undecidability of the two quantifier theory in the language extended by symbols for both join and infimum with the understanding that the result applies to any total extension of the infimum relation on $\mathcal{D}_{T}$ which, by Kleene and Post [1954], is not a lattice. (This proof also supplies another one for the undecidability of the three quantifier theory in the language with just $\leq_{T}$.)

The same results on embedding as partial orderings and initial segments, and so the corresponding applications to the analysis of their theories, can be proved by quite analogous types of constructions (Cohen and perfect tree forcing) for a range of reducibilities from truth table to arithmetic, $A \leq_{a} B \Leftrightarrow A \leq_{T} B^{(n)}$ for some $n \in \omega$, (see e.g. Feferman [1965], Nerode and Shore [1980], Odifreddi [1983], M. Simpson [1985]). When one moves from the realm of recursion theory and the natural numbers to set theory and the ordinals, new issues arise.

If we look, for example, at relative constructibility $\left(A \leq_{c} B \Leftrightarrow A \in L[B]\right.$ for $\left.A, B \subseteq \omega\right)$ and the constructibility degrees, $\mathcal{D}_{c}$, of subsets of $\omega$, we are faced with the new problem of preserving $\omega_{1}$ in our forcing extensions. For Cohen forcing this was part of Cohen's seminal results and one can carry over (under suitable set theoretic hypotheses which we ignore here) the partial order embedding (Cohen [1966]) and related results to $\leq_{c}$ with little difficulty (see, e.g. Balcar and Hajek [1978], Farrington [1983], [1984] and Abraham and Shore [1986a]). Our ability to preserve $\omega_{1}$ for perfect forcing is due to Sacks [1971] and is based on what he calls the fusion lemma. It enabled Sacks [1971] to prove the existence of a minimal degree of constructibility.

The next major steps towards determining the initial segments of $\mathcal{D}_{c}$ were taken by Adamowicz who proved first [1976] that all finite lattices can be embedded as initial segments of $\mathrm{D}_{c}$ and then [1977] that all countable constructible well founded usls can be so embedded. The extra assumptions Adamowicz needed for her proof turned out to be to some extent necessary. Abraham and Shore [1986a] showed that not every countable well founded distributive lattice is isomorphic to an initial segment of $\mathcal{D}_{c}$. The crucial fact here is that the relation $\leq_{c}$ is itself constructible and so one can get 
the result by coding nonconstructible sets in distributive lattices. Even if we restrict attention to constructible lattices, some remnants of the well foundedness assumption remain necessary. Lubarsky [1987] proved that every countable lattice isomorphic to an initial segment of $\mathcal{D}_{c}$ is complete. On the positive side, the best results are those of Groszek and Shore [1988] that every countable (dual) algebraic lattice $\mathcal{L}$ (i.e. $\mathcal{L}$ is complete and generated by its compact elements $x$ for which $\wedge I \leq x$ implies that there is a finite $F \subseteq I$ such that $\wedge F \leq x$, for every $I \subseteq \mathcal{L}$ ). (There is new interesting work, however, by Dorais [2007] on the c-degrees of subsets of $\aleph_{1}$ produced by forcing with Souslin trees instead of countable perfect trees.)

Our concern in this paper is a reducibility and degree notion lying between that of arithmetic and constructible: the hyperarithmetic degrees $\mathcal{D}_{h}$. For Turing reducibility and $\mathcal{D}_{T}$ and arithmetic reducibility and $\mathcal{D}_{a}$, the setting is first order arithmetic and the natural numbers or $\omega$. For set theory we have relative constructibility and $\omega_{1}$. Our setting is second order arithmetic and $\omega_{1}^{C K}$, the first nonrecursive ordinal. It takes the place of $\omega_{1}$, the first uncountable ordinal, as its effective analog. We say that $A$ is hyperarithmetic in $B, A \leq_{h} B$, if $A \leq_{T} B^{(\alpha)}$ for some ordinal $\alpha<\omega_{1}^{B}$, i.e. the order type of $\alpha$ has a representative recursive in $B$. Here $B^{(\alpha)}$ is the $\alpha^{\text {th }}$ iterate of the Turing jump applied to $B$ where effective (in $B$ ) unions are taken at limit levels. For another view, note that Kleene showed (see Sacks [1990, II.1-2]) that $A \leq_{h} B$ if and only if $A$ is $\Delta_{1}^{1}(B)$.

In this setting, Feferman [1965] (see also Sacks [1990]) introduced a ramified language for second order arithmetic and the appropriate notion of Cohen forcing. He proved (among other results) that every countable partial order is embeddable in $\mathcal{D}_{h}$ (even below the hyperarithmetic degree of Kleene's $\mathcal{O}$, the complete $\Pi_{1}^{1}$ set). Some extensions and related results using Cohen forcing are in Thomason [1967] and [1969] and also Odifreddi [1983a]. The crucial fact needed is the preservation of $\omega_{1}^{C K}$, i.e. if $G$ is Cohen generic in this setting then $\omega_{1}^{G}=\omega_{1}^{C K}$. (This turns out to be equivalent to $\Delta_{1}^{1}$-comprehension holding in the generic extension.)

One can also adapt perfect forcing to this setting to construct a minimal hyperdegree (Gandy and Sacks [1967] or with more detailed exposition and explanation Sacks [1971] or [1990]). Again the crucial issue is the preservation of $\omega_{1}^{C K}$ (or $\Delta_{1}^{1}$-comprehension) by a fusion lemma (see $\S 3$ below). Here delicate definability issues and the theory of $\Pi_{1}^{1}$ sets play a role not seen in either the arithmetic or set theoretic settings. In contrast to all the other degree structures discussed so far, almost nothing more has been known about initial segments of $\mathcal{D}_{h}$.

Thomason [1970] proved that every finite distributive lattice is isomorphic to an initial segment of $\mathcal{D}_{h}$ and there matters stood. In his review of this paper, Sacks [1972] writes as follows:

"He raises a methodologically interesting open question: is every countable distributive lattice isomorphic to an initial segment of hyperdegrees? The answer (according to the reviewer) is probably yes, but (as the author points out) the method of the paper does not suffice to prove it." 
Now in 1970 Lerman's methods for handling nondistributive lattices were not yet available but for the distributive ones there was as much available then as now. Thus the methodological issues were not about the initial segment constructions as used in $\mathcal{D}_{T}$ but rather about making the analog of the fusion lemma work in more general settings. Constructing embeddings of infinite lattices required (even in the later Turing degree constructions) imposing more and more restrictions on the trees as the construction progressed (often using finite approximations to the lattice to guide them). This type of forcing condition is not amenable to the arguments for the fusion lemma as the nature of the conditions change as more elements of the lattice are considered and so fusing an infinite sequence of such conditions produces a tree that is not a condition. The argument for the constructibility degrees gets around this problem by restricting attention to constructible lattices so that the entire lattice can be treated at once and so uses trees of a single shape just with branchings that grow to match the uniformly constructible approximations to the given lattice. One can then prove the set theoretic fusion lemma to preserve $\omega_{1}$ much as for binary trees.

This approach would work for the hyperdegrees as well but would be restricted to (dual) algebraic hyperarithmetical lattices and so, of course, it would not suffice to embed all the distributive lattices. Now for the constructibility degrees some such restrictions were necessary and not all countable distributive lattices (or even linear orders) are isomorphic to initial segments of $\mathcal{D}_{c}$. This analogy makes it seem less likely that Sacks's conjecture about the initial segments of $\mathcal{D}_{h}$ is true especially since all the available techniques seem to be quite similar.

One thus turns to finding counterexamples. The crucial fact used both in the first examples of distributive lattices not isomorphic to initial segments of $\mathcal{D}_{c}$ and in the later proofs of the necessity of completeness was that $\leq_{c}$ is itself a constructible relation. For the Turing degrees, $\leq_{T}$ is far from recursive: it is only a $\Sigma_{3}^{0}$ relation. The hyperdegrees lie in between (in this sense as well) as $\leq_{h}$ is a $\Pi_{1}^{1}$ relation and so analogous to $\Sigma_{1}^{0}$ or r.e. ones in the setting of the Turing degrees. $\left(A \leq_{h} B\right.$ if and only if both $A$ and its complement are $\Pi_{1}^{1}$ in $B$.) Thus the construction of even the basic counterexamples requires more delicacy. We here use the methods of finitely generated successor models introduced in Shore [1981] and used in the setting of $\mathcal{D}_{h}$ in Shore [2007], [2008] to prove Slaman and Woodin's result that $\mathcal{D}_{h}$ is rigid and biinterpretable with second order arithmetic by purely degree theoretic arguments that work locally (in jump ideals) rather than just in $\mathcal{D}_{h}$ as a whole.

As every initial segment of $\mathcal{D}_{h}$ (or any of our degree structures) has a least element we assume that all (upper or lower semi)lattices have a least element 0. As we only consider countable lattices, we also consider only (upper or lower semi)lattices with a greatest element, 1, as well. We think of both 0 and 1 as named by constants in the language and so are preserved under substructures and extensions, even as upper or lower semilattices.

Theorem 1.1. Not every countable lattice is isomorphic to an initial segment of the hyperdegrees. 
Proof. Shore [2007, §2] and [2008, §2-3] present a method for taking any set $X$ and constructing a lattice $\mathcal{L}_{X}$ such that if $f$ is an embedding of $\mathcal{L}_{X}$ into $\mathcal{D}_{h}$ then $X \leq_{h} f\left(0_{\mathcal{L}_{X}}\right)$. Thus if we take $X$ to be, for example, Kleene's $\mathcal{O}$ or any nonhyperarithmetic set, any embedding $f$ of $\mathcal{L}_{X}$ as an initial segment of $\mathcal{D}_{h}$ would contradict the assumption that $X$ is not hyperarithmetic.

We defer a more detailed explanation of this coding method to $\S 6$ where we need an elaboration for a finer result. For now it suffices to state that the lattices constructed contain finitely many elements which generate (using $\vee$ and $\wedge$ ) a sequence of incomparable elements of type $\omega$ and other parameters that define the subsets of this sequence corresponding to $X$ and its complement. Moreover, the recovery procedure producing $X$ (and its complement) is positively $\Sigma_{1}^{0}$ in the partial order relation and join operator of the lattice and so $\Pi_{1}^{1}$ (and so $\Delta_{1}^{1}$ ) in (by an additional trick) the bottom (and not just top) set of any embedding. Thus these lattices have two seemingly crucial properties that would lead to our contradiction if they could be realized as initial segments of $\mathcal{D}_{h}$. The first is that the lattice is not hyperarithmetic. This is analogous to the first examples in $\mathcal{D}_{c}$ except that the coding is more delicate and survives $\leq_{h}$ being only $\Pi_{1}^{1}$ rather than hyperarithmetic. On the other hand, this same difference makes the direct coding methods into distributive lattices used for $\mathcal{D}_{c}$ unavailable in $\mathcal{D}_{h}$. The second aspect of the argument is that the coding (of nonhyperarithmetic information) used here seems to rely on the fact that it is finitely generated as it uses the generators as parameters in the decoding. This type of coding cannot be carried out in distributive lattices as they are all locally finite lattices: every finite subset generates a finite sublattice. This obstacle to constructing counterexamples then revives the possibility of verifying Sacks's conjecture for distributive lattices.

In fact, we prove that both of these properties (nonhyperarithmetic and not locally finite) are necessary for a countable lattice not to be isomorphic to an initial segment of $\mathcal{D}_{h}$. Perhaps surprisingly, we provide a common generalization of a lattice being either hyperarithmetic or locally finite.

Theorem 1.2. Every sublattice $\mathcal{K}$ of any hyperarithmetic lattice $\mathcal{L}$ is isomorphic to an initial segment of the hyperdegrees. In fact, it can be realized as an initial segment with top a hyperdegree below that of $\mathcal{O} \oplus \mathcal{K}$.

To see that this is indeed the desired common generalization we need to show that every countable locally finite lattice is isomorphic to a sublattice of a hyperarithmetic lattice.

Proposition 1.3. There is a recursive, universal, locally finite lattice, i.e. a recursive, locally finite lattice into which every countable, locally finite lattice can be embedded.

Proof. With the proper organization of the requirements, the standard Fraissé construction (as in Hodges [1993, 7.1.2] produces the desired lattice once one has the amalgamation property for the class of finite lattices. 
Lemma 1.4. The class of finite lattices (with 0 and 1 ) has the amalgamation property, i.e. if $\mathcal{A}, \mathcal{B}_{0}$ and $\mathcal{B}_{1}$ are finite lattices and $f_{0}, f_{1}$ are embeddings of $\mathcal{A}$ into $\mathcal{B}_{0}$ and $\mathcal{B}_{1}$, respectively, then there is a finite lattice $\mathcal{C}$ and embeddings $g_{0}$ and $g_{1}$ of $\mathcal{B}_{0}$ and $\mathcal{B}_{1}$, respectively, into $\mathcal{C}$ such that $g_{0} f_{0}\left\lceil\mathcal{A}=g_{1} f_{1}\lceil\mathcal{A}\right.$.

Proof. This should be a known fact but we have not found a reference to the precise form of the amalgamation property that we need. We supply a proof at the end of $\S 5$.

As we mentioned above, every distributive lattice is locally finite (this is well known and follows easily from the Stone Representation Theorem (see e.g. Grätzer [2003, p. 85]) that it is isomorphic to a ring of sets). Thus we have our answer to the original question of Thomason [1970] and Sacks [1972].

Corollary 1.5. Every locally finite and so, in particular, every countable distributive lattice is isomorphic to an initial segment of the hyperdegrees.

These results also allow us to establish the same fine line between decidability and undecidability in the fragments of the theory of $\mathcal{D}_{h}$ as one has for $\mathcal{D}_{T}$.

Theorem 1.6. The two quantifier theory of $\mathcal{D}_{h}$ is decidable while the three quantifier theory is undecidable as is the two quantifier theory in the language with both $\vee$ and $\wedge$ where $\wedge$ denotes any total extension of the infimum relation.

Proof. The proofs for the first two assertions in $\mathcal{D}_{T}$ (see Lerman [1983 VII.4, II.4.11 and A.2.9] although some minor corrections are needed) rely only on two facts. First, the structure is an usl with 0 in which all finite lattices can be embedded as initial segments. Second, it requires a Kleene-Post type extension of embedding theorem: Given any finite usl $P$ extended as a partial order by a finite $Q$ and any embedding of $P$ into $\mathcal{D}_{T}$ there is an extension of the embedding to one of $Q$ as long as $Q$ respects the usl structure of $P$ and has no new elements below any of those in $P$. Our results supply the first fact. Cohen forcing in the hyperarithmetic setting supplies the second. (The required extension of the given embedding can easily be constructed from a set of hyperdegrees independent over all the given degrees in the image of $P$ as in the standard construction of an embedding of an arbitrary partial order. Such independent degrees are given by the columns of a Cohen generic set (in the hyperarithmetic sense) by relativizing Feferman [1965] to the top given degree.) The final undecidability result needs only that all recursive lattices are isomorphic to initial segments as in Miller, Nies and Shore [2003, Cor. 3.2].

The crucial aspect of our version of perfect trees (and associated forcing notion) that allows us to prove the required fusion lemma is a type of forgetfulness property (Proposition 3.2). We will use pairs consisting of trees $T$ and a finite sublowersemilattice $(s l s l) \hat{\mathcal{K}}$ of $\mathcal{L}$. The shape of the tree is determined in advance by a type of sequential usl representation for $\mathcal{L}$ and the second component indicates the congruence relations that must be respected in all further refinements. (All these notions are made precise 
in $\S 2$.) What goes wrong in a naive attempt to transfer the methods of either $\mathcal{D}_{T}$ or $\mathcal{D}_{c}$ is the variation in the congruences that must be respected. Our forcing will have the property that if some condition forces a sentence of a certain form then the second component is irrelevant and so can be made the same in all the extensions needed in the fusion lemma. While this may seem unlikely, it is possible once one has proven a new lattice representation theorem (Theorem 5.1). Its construction takes into account various requirements for all sublattices at every step. The full lattice structure is exploited here by using a slsl decomposition of $\mathcal{L}$ and usl representations for the slsls. (The slsls are actually lattices as they are finite although with elements having possibly different joins than in all of $\mathcal{L}$.)

While all of this could have been done in the setting of $\mathcal{D}_{T}$ (and actually gives some simplified proofs for various cases) no new theorems, of course, can be proven this way. On the other hand, given the known counterexamples in $\mathcal{D}_{c}$, it is clear that the methods cannot carry over to that setting. Surprisingly, all the recursion theoretic arguments can be carried over and all the uses of fusion other than to preserve $\omega_{1}^{C K}$ can be avoided. (Those used to show that deciding each sentence is dense are, of course, unnecessary. Those to convert reductions to ones resembling Turing ones can be replaced by local Cohen forcing inside the tree parts of the conditions.) Thus it must be that the forcing notion in the set theoretic case analogous to ours, but using constructible trees instead of hyperarithmetic ones, fails to preserve $\omega_{1}$ despite the fact that ours preserves $\omega_{1}^{C K}$.

Before beginning the detailed proofs of our results, we also want to make a comment on the coding methods used. While early results using codings in degree structures in general (and later ones for $\mathcal{D}_{c}$ ) used distributive lattices, later stronger ones have all used nondistributive ones. It turns out that we now have a formal proof of the necessity of moving to nondistributive lattices to get the best results. While one can code arbitrary sets into distributive lattices (e.g. by the venerable lines and diamonds method) the decoding takes a number of quantifiers. In degree structures, however, while join is an effective operation (on indices) meet is not and requires extra quantifiers. Thus to get the best possible results one wants a coding for which the decoding is r.e. in only $\leq$ and $\checkmark$ (with only positive occurrences of $\leq$ as negative ones also add to the complexity). This is the type of coding used in the many applications of effective successor models from Shore [1981] to [2008]. As explicitly stated in Shore [2007] and [2008] one has a recursive sequence $\phi_{n}$ of positive $\Sigma_{1}$ formulas in $\leq$ and $\vee$ such that given any set $X$ there is a countable lattice $\mathcal{L}_{X}$ (even one recursive in $X$ ) such that $n \in X \Leftrightarrow \mathcal{L}_{X} \models \phi_{n}$. Our results show that there is no such coding procedure in distributive lattices. Indeed, there is an $X$ (actually the complement of $\mathcal{O}$ ) such that there is no hyperarithmetic sequence $\phi_{n}$ of positive $\Sigma_{1}$ formulas in $\leq$ and $\vee$ and no distributive (or even locally finite) countable lattice $\mathcal{L}_{X}$ such that $n \in X \Leftrightarrow \mathcal{L}_{X} \models \phi_{n}$. To see this, suppose there were such an $\mathcal{L}$. Take an embedding of $\mathcal{L}$ as an initial segment of $\mathcal{D}_{h}$ with top the degree of some $G$. As $\leq_{h}$ and $\vee$ are $\Pi_{1}^{1}(G)$ on (the indices of) the initial segment of $\mathcal{D}_{h}$ below $\operatorname{deg}_{h}(G)$, $X$, the complement of $\mathcal{O}$ would be $\Pi_{1}^{1}$ in $G$ and so $\mathcal{O}$ would be hyperarithmetic in $G$ but the hyperdegrees below $\mathcal{O}$ do not form a lattice. On the other hand, our coding 
in Proposition 6.2 shows that there is such a recursive sequence $\phi_{n}$ such that given any $X$ we can find an usl $\mathcal{K}_{X}$ which is a susl of a locally finite lattice $\mathcal{L}_{X}$ (each recursive in $X$ ) such that $n \in X \Leftrightarrow \mathcal{K}_{X} \models \phi_{n}$. In a slightly different vein, it seems that using the coding in distributive lattices found in Selivanov [1988] one can code arbitrary sets into distributive lattices in this way if one gives up the requirement that the sentences $\phi_{n}$ are positive. (Of course, this would increase the absolute complexity of the decoding procedure for $\mathcal{D}_{T}$ or $\mathcal{D}_{h}$.)

Our plan now is to present the definitions of usl representations and our forcing notion in $\S 2$. We also prove some basic facts and state the representation theorem we need. The actual proof of the theorem we need is given in $\S 5$. The crucial fusion lemma is proven in $\S 3$ while the lemmas needed for the construction of our generic sequence and the verifications that it gives the desired initial segment of $\mathcal{D}_{h}$ are in $\S 4$. Finally, we close in $\S 6$ with a discussion of the situation for countable usl initial segments of $\mathcal{D}_{h}$ and various open questions.

\section{Usl representations and the notion of forcing}

We are given a hyperarithmetic lattice $\mathcal{L}$ and a sublattice $\mathcal{K}$ (both with the same 0 and 1). We begin our journey to the required notion of forcing with the definition of an uppersemilattice (usl) representation.

Definition 2.1. Let $\Theta$ be a set of maps from an usl $\mathcal{L}$ into $\omega$. For $\alpha, \beta \in \Theta$ and $x \in \mathcal{L}$, we write $\alpha \equiv_{x} \beta$ ( $\alpha$ is congruent to $\beta$ modulo $x$ ) if $\alpha(x)=\beta(x)$. We write $\alpha \equiv_{x, y} \beta$ to indicate that $\alpha$ is congruent to $\beta$ modulo both $x$ and $y$ and generally use commas conjunctively in this way. Such a $\Theta$ is an usl representation of $\mathcal{L}$ if it contains the function that is 0 on every input and for every $\alpha, \beta \in \Theta$ and $x, y, z \in \mathcal{L}$ the following properties hold:

1. $\alpha(0)=0$.

2. (Differentiation) If $x \not \leq y$ then there are $\gamma, \delta \in \Theta$ such that $\gamma \equiv_{y} \delta$ but $\gamma \not \equiv_{x} \delta$.

3. (Order) If $x \leq y$ and $\alpha \equiv_{y} \beta$ then $\alpha \equiv_{x} \beta$.

4. (Join) If $x \vee y=z$ and $\alpha \equiv_{x, y} \beta$ then $\alpha \equiv_{z} \beta$.

Notation 2.2. If $\Theta$ is an usl representation for $\mathcal{L}$ and $\hat{\mathcal{L}} \subseteq \mathcal{L}$ then we denote the restriction of $\Theta$ to $\hat{\mathcal{L}}$ by $\Theta \uparrow \hat{\mathcal{L}}=\{\alpha \uparrow \hat{\mathcal{L}} \mid \alpha \in \Theta\}$. We also say that $\Theta$ is an extension of $\Theta \uparrow \hat{\mathcal{L}}$.

For those familiar with lattice representations as presented in lattice theory we note that these representations (with the additional requirements for infimum given below in Theorem $5.1(2))$ essentially correspond to ones of the dual lattice by equivalence 
relations. If the reader prefers to think in terms of equivalence relations on a set then the set is $\Theta$, the relation that corresponds to $x \in \mathcal{L}$ is $\equiv_{x}$ and, for $\alpha \in \Theta$, its $x$-equivalence class is $\alpha(x)$. A further reduction making the set $\Theta$ into one of natural numbers can be achieved by identifying the elements $\alpha$ of $\Theta$ with their values at the 1 of $\mathcal{L}$ (and so some natural number $\alpha(1)$ ) as this uniquely determines all the values of $\alpha$ by the order property. We find this last identification that views the elements of $\mathcal{L}$ and those of $\Theta$ as of the same type to be confusing. (It is really the equivalence classes that correspond to the elements of $\mathcal{L}$.) We also find the functional notation convenient for the construction of representations in $\S 5$.

Definition 2.3. If $\Theta^{\prime}$ and $\Theta$ are usl representations for $\mathcal{L}^{\prime}$ and $\mathcal{L}$, respectively, $\hat{\mathcal{L}} \subseteq \mathcal{L}^{\prime} \subseteq \mathcal{L}$ and $f: \Theta^{\prime} \rightarrow \Theta$, then $f$ is an $\hat{\mathcal{L}}$-homomorphism if, for all $\alpha, \beta \in \Theta^{\prime}$ and $x \in \hat{\mathcal{L}}$, $\alpha \equiv_{x} \beta \Rightarrow f(\alpha) \equiv_{x} f(\beta)$.

In $\S 5$ we will prove the existence of a special type of representation for our given lattice $\mathcal{L}$ that we need to define our forcing conditions.

Theorem 5.1. If $\mathcal{L}$ is a countable lattice then there is an usl representation $\Theta$ of $\mathcal{L}$ along with a nested sequence of finite slsls $\mathcal{L}_{i}$ starting with $\mathcal{L}_{0}=\{0,1\}$ with union $\mathcal{L}$ and a nested sequence of finite subsets $\Theta_{i}$ with union $\Theta$ with both sequences recursive in $\mathcal{L}$ with the following properties:

1. For each $i, \Theta_{i}\left\lceil\mathcal{L}_{i}\right.$ is an usl representation of $\mathcal{L}_{i}$.

2. There are meet interpolants for $\Theta_{i}$ in $\Theta_{i+1}$, i.e. if $\alpha \equiv_{z} \beta, x \wedge y=z$ (with $\alpha, \beta \in \Theta_{i}$ and $\left.x, y, z \in \mathcal{L}_{i}\right)$ then there are $\gamma_{0}, \gamma_{1}, \gamma_{2} \in \Theta_{i+1}$ such that $\alpha \equiv_{x} \gamma_{0} \equiv_{y} \gamma_{1} \equiv_{x} \gamma_{2} \equiv$ $\beta$.

3. For every sublowersemilattice $\hat{\mathcal{L}}$ of $\mathcal{L}_{i}, \hat{\mathcal{L}} \subseteq_{l s l} \mathcal{L}_{i}$, there are homogeneity interpolants for $\Theta_{i}$ with respect to $\hat{\mathcal{L}}$ in $\Theta_{i+1}$, i.e. for every $\alpha_{0}, \alpha_{1}, \beta_{0}, \beta_{1} \in \Theta_{i}$ such that $\forall w \in \hat{\mathcal{L}}\left(\alpha_{0} \equiv_{w} \alpha_{1} \rightarrow \beta_{0} \equiv_{w} \beta_{1}\right)$, there are $\gamma_{0}, \gamma_{1} \in \Theta_{i+1}$ and $\hat{\mathcal{L}}$-homomorphisms $f, g, h: \Theta_{i} \rightarrow \Theta_{i+1}$ such that $f: \alpha_{0}, \alpha_{1} \mapsto \beta_{0}, \gamma_{1}, g: \alpha_{0}, \alpha_{1} \mapsto \gamma_{0}, \gamma_{1}$ and $h: \alpha_{0}, \alpha_{1} \mapsto \gamma_{0}, \beta_{1}$, i.e. $f\left(\alpha_{0}\right)=\beta_{0}, f\left(\alpha_{1}\right)=\gamma_{1}$ etc.

We point out that the presentations in Lerman [1983] and Lachlan and Lebeuf [1976] are phrased in terms of there being only one element/two functions homogeneity interpolants instead of the two/three we required in our definition. One/two are not sufficient in general and two/three are used in the original proof for finite lattices in Lerman [1971] and ones of size $\aleph_{1}$ in Abraham and Shore [1986]. In addition, we have changed the definition of homogeneity interpolants from the one used in Lerman [1971] and elsewhere in the literature by requiring that $g: \alpha_{0}, \alpha_{1} \longmapsto \gamma_{0}, \gamma_{1}$ (in place of $\gamma_{1}, \gamma_{0}$ ). This variation is a different special case than Lerman's of a more general definition introduced in KjosHanssen [2002], [2003]. (Kjos-Hanssen's version allows a finite sequence of interpolants $\gamma_{i}$ and functions $f_{i}$ and is indifferent to order in that sense that it only requires that 
$\left\{f_{i}\left(\alpha_{0}\right), f_{i}\left(\alpha_{1}\right\}\right\}=\left\{\gamma_{i}, \gamma_{i+1}\right\}$.) Adopting our version does not makes the construction of the required interpolants (Proposition 5.6) any more difficult but makes our construction of the crucial splitting trees in Proposition 4.5 considerably simpler than the previous ones. (The same simplification would carry over to $\mathcal{D}_{T}$ and other degree structures.) All the previous constructions proceed by using only susls and usl representations. We have mixed in the full lattice structure by requiring the decomposition of $\mathcal{L}$ to be into slsls instead while still using usl representations. We exploit the full lattice structure at various points in our construction. We will see, moreover, in $\S 6$ that this use of the meet structure of $\mathcal{L}$ is also necessary (in contrast to the situation in $\mathcal{D}_{T}$ ). Given such $\mathcal{L}_{i}$ and $\Theta_{i}$ we next need to define the class of trees that will be eligible to be the first component of our forcing conditions. Each of our trees will be a function from finite strings to finite strings (of elements from the $\Theta_{i}$ ) with various properties.

Definition 2.4. A tree $T$ (for the sequence $\left\langle\mathcal{L}_{i}, \Theta_{i}\right\rangle$ ) has an associated natural number $k=k(T)$. $T$ is a hyperarithmetic function such that for some $k \in \omega$ it has the empty string $\emptyset$ and all strings in the Cartesian product $\prod_{n=0}^{n=m} \Theta_{k+n}$ for each $m \in \omega$. We denote this number $k$ by $k(T)$. Moreover, for each $\sigma \in \operatorname{dom} T, T(\sigma) \in \prod_{n=0}^{n=q} \Theta_{n}$ for some $q \geq|\sigma|-1$. Moreover, $T$ has the following properties for all $\sigma, \tau \in \operatorname{dom} T$ :

1. (Order) $\sigma \subseteq \tau \Rightarrow T(\sigma) \subseteq T(\tau)$.

2. (Nonorder) $\sigma|\tau \Rightarrow T(\sigma)| T(\tau)$. (We use $\mid$ to denote incompatibility of strings.)

3. (Uniformity) For every fixed length $l$ there is, for each $\alpha \in \Theta_{k+l}$, a string $\rho_{l, \alpha}$ so that, for a given $l$, all the $\rho_{l, \alpha}$ are of the same length independently of $\alpha$ and if $|\sigma|=l$ then $T\left(\sigma^{\wedge} \alpha\right)=T(\sigma)^{\wedge} \rho_{l, \alpha}$. (We use ^ to denote concatenation and confuse a single symbol such as $\alpha$ with the string $\langle\alpha\rangle$ of length one.)

Thus our trees $T$ have branchings of width $\left|\Theta_{k(T)+n}\right|$ at level $n$ and obey the usual order and uniformity properties of those used in Lerman [1983] to prove initial segment results for $\mathcal{D}_{T}$. Our definition of $S$ being a subtree of $T$ incorporates the (technically convenient) requirement also used there that the branchings on $S$ follow those on $T$.

Definition 2.5. We say that a tree $S$ is a subtree of a tree $T, S \subseteq T$, if $k(S) \geq k(T)$ and $(\forall \sigma \in \operatorname{dom} S)(\exists \tau \in \operatorname{dom} T)\left[S(\sigma)=T(\tau) \&\left(\forall \alpha \in \Theta_{k(S)+|\sigma|}\right)\left(S\left(\sigma^{\wedge} \alpha\right) \supseteq T\left(\tau^{\wedge} \alpha\right)\right)\right]$.

If it does not seem obvious, transitivity of the subtree relation will be proven in Proposition 2.8. We mention some specific operations on trees that we will need later.

Definition 2.6. If $T$ is a tree and $\sigma \in \operatorname{dom} T$ then $T_{\sigma}$ is defined by $T_{\sigma}(\tau)=T\left(\sigma^{\wedge} \tau\right)$. Clearly, $k\left(T_{\sigma}\right)=k(T)+|\sigma|$ and $T_{\sigma} \subseteq T$. For a string $\mu \in \prod_{n=0}^{n=q} \Theta_{n}$ with $q \leq|T(\emptyset)|-1$, we 
let $T^{\mu}$ (the transfer tree of $T$ over $\mu$ ) be the tree such that, for every $\sigma \in \operatorname{dom} T, T^{\mu}(\sigma)$ is the string gotten from $T(\sigma)$ by replacing its initial segment of length $q+1$ (which is contained in $T(\emptyset))$ by $\mu$. We write $T_{\sigma}^{\mu}$ for $\left(T_{\sigma}\right)^{\mu}$.

A crucial notion for our constructions is that of preserving the congruences of specified slsls of our given lattice $\mathcal{L}$.

Definition 2.7. If $\hat{\mathcal{L}}$ is a finite slsl of $\mathcal{L}$ we say that a subtree $S$ of $T$ preserves the congruences of $\hat{\mathcal{L}}, S \subseteq \subseteq_{\hat{\mathcal{L}}} T$, if $\hat{\mathcal{L}} \subseteq \mathcal{L}_{k(T)}$ and, whenever $x \in \hat{\mathcal{L}}, S(\sigma)=T(\tau), \alpha \equiv_{x} \beta$, $S\left(\sigma^{\wedge} \alpha\right)=T\left(\tau^{\wedge} \mu\right)$ and $S\left(\sigma^{\wedge} \beta\right)=T\left(\tau^{\wedge} \nu\right)$, then $\mu \equiv_{x} \nu$. Here $\alpha$ and $\beta$ are members of the appropriate $\Theta_{i}$ and $\mu$ and $\nu$ are sequences (necessarily of the same length $m$ ) of elements from the appropriate $\Theta_{j}$ 's. We say that such sequences $\mu$ and $\nu$ are congruent modulo $x, \mu \equiv_{x} \nu$, if $\mu(j) \equiv_{x} \nu(j)$ for each $j<m$.

Proposition 2.8. If $R \subseteq_{\mathcal{L}_{1}} S \subseteq_{\mathcal{L}_{2}} T$ and then $R \subseteq_{\mathcal{L}_{1} \cap \mathcal{L}_{2}} T$.

Proof. To see that $R \subseteq T$ note first that $k(R) \geq k(S) \geq k(T)$. Next suppose that $\rho \in \operatorname{dom} R$ and $\alpha \in \Theta_{k(R)+|\rho|}$. As $R \subseteq S$ we have a $\sigma$ such that $R(\rho)=S(\sigma)$ and $\left.R\left(\rho^{\wedge} \alpha\right) \supseteq S\left(\sigma^{\wedge} \alpha\right)\right)$. As $S \subseteq T$ we have a $\tau$ such that $S(\sigma)=T(\tau)$ and $\left.S\left(\sigma^{\wedge} \alpha\right) \supseteq T\left(\tau^{\wedge} \alpha\right)\right)$. Thus $R(\rho)=T(\tau)$ and $R\left(\rho^{\wedge} \alpha\right) \supseteq T\left(\tau^{\wedge} \alpha\right)$ as required. As for the preservation of $\mathcal{L}_{1} \cap \mathcal{L}_{2}$ congruences, suppose $R(\rho)=S(\sigma)=T(\tau), x \in \mathcal{L}_{1} \cap \mathcal{L}_{2}, \alpha_{0}, \alpha_{1} \in \Theta_{k(R)+|\rho|}$ and $\alpha_{0} \equiv_{x} \alpha_{1}$. Let $R\left(\rho^{\wedge} \alpha_{i}\right)=S\left(\sigma^{\wedge} \mu_{i}\right)=T\left(\tau^{\wedge} \nu_{i}\right)$. As $x \in \mathcal{L}_{1}$ and $R \subseteq_{\mathcal{L}_{1}} S, \mu_{0} \equiv_{x} \mu_{1}$. As $x \in \mathcal{L}_{2}$ and $S \subseteq \subseteq_{\mathcal{L}_{2}} T$ it then follows by induction on the (by uniformity, necessarily common) length of $\mu_{i}$ that $\nu_{0} \equiv_{x} \nu_{1}$ as required. (Write $\nu_{i}=\nu_{i}^{0 \wedge} \cdots^{\wedge} \nu_{i}^{s}$ where $S\left(\sigma^{\wedge} \mu_{i}(0) \cdots{ }^{\wedge} \mu_{i}(t)\right)=$ $T\left(\tau^{\wedge} \nu_{i}^{0 \wedge \cdots \wedge} \nu_{i}^{t}\right)$. Then inductively $\mu_{0}(j) \equiv_{x} \mu_{1}(j)$ gives $\nu_{0}^{j} \equiv_{x} \nu_{1}^{j}$.)

Definition 2.9. Recall that $\mathcal{K}$ is an arbitrary sublattice of $\mathcal{L}$ (preserving 0 and 1 ). Our notion of forcing $\mathcal{P}_{\left\langle\mathcal{L}_{i}, \Theta_{i}\right\rangle, \mathcal{K}}=\mathcal{P}$ is given first by letting the forcing conditions $P$ be the pairs $\langle T, \hat{\mathcal{K}}\rangle$ where $T$ is a tree for $\left\langle\mathcal{L}_{i}, \Theta_{i}\right\rangle$ and $\hat{\mathcal{K}}$ is a finite slsl of $\mathcal{K} \cap \mathcal{L}_{k(T)}$. We then say that $\left\langle T_{1}, \mathcal{K}_{1}\right\rangle$ extends or refines $\left\langle T_{0}, \mathcal{K}_{0}\right\rangle,\left\langle T_{1}, \mathcal{K}_{1}\right\rangle \leq_{\mathcal{P}}\left\langle T_{0}, \mathcal{K}_{0}\right\rangle$ if $T_{1} \subseteq \mathcal{K}_{0} T_{0}$ and $\mathcal{K}_{1} \supseteq \mathcal{K}_{0}$. If $P=\langle T, \hat{\mathcal{K}}\rangle$ is a condition we let $K(P)=\hat{\mathcal{K}}, \operatorname{Tr}(P)=T$ and $k(P)=k(T)$. If the notion of forcing is fixed in some context we will often omit the subscript $\mathcal{P}$ in $\leq_{\mathcal{P}}$. We sometimes abuse notation by identifying a condition $P$ with $\operatorname{Tr}(P)$ when $K(P)$ is fixed. Along these lines, for example, we use $P_{\sigma}, P^{\tau}$ and $P_{\sigma}^{\tau}$ to stand for $\left\langle\operatorname{Tr}(P)_{\sigma}, K(P)\right\rangle,\left\langle\operatorname{Tr}(P)^{\tau}, K(P)\right\rangle$ and $\left\langle\operatorname{Tr}(P)_{\sigma}^{\tau}, K(P)\right\rangle$, respectively. The top element of $\mathcal{P}$ consists of the identity tree $I d$ (which has $k(I d)=0$ ) and the slsl $\mathcal{L}_{0}=\{0,1\}$.

Lemma 2.10. If $T$ is a tree, $\sigma \in \operatorname{dom} T$ and $\hat{\mathcal{L}} \subseteq \mathcal{L}_{k(T)}$, then $T_{\sigma} \subseteq \subseteq_{\mathcal{L}} T$. So if $P=\langle T, \hat{\mathcal{K}}\rangle$ is a condition $\left\langle T_{\sigma}, \hat{\mathcal{K}}\right\rangle \leq_{\mathcal{P}}\langle T, \hat{\mathcal{K}}\rangle$ (by letting $\hat{\mathcal{L}}=\hat{\mathcal{K}}$ ). If $\sigma, \tau \in$ dom $T$ are of the same length and $\langle S, \hat{\mathcal{K}}\rangle \leq\left\langle T_{\sigma}, \hat{\mathcal{K}}\right\rangle$ then $\left\langle S^{T(\tau)}, \hat{\mathcal{K}}\right\rangle \leq_{\mathcal{P}}\left\langle T_{\tau}, \hat{\mathcal{K}}\right\rangle$. We also have that $T_{\sigma}^{T(\tau)}=T_{\tau}$.

Proof. The first assertions follow directly from the definitions. The last two follow from the uniformity assumption on our trees. 
It is easy to see that with trivial genericity requirements any generic filter $\mathcal{G}$ determines a function $G \in D=\prod_{n=0}^{\infty} \Theta_{n}$, i.e. a function on $\omega$ with $G(n) \in \Theta_{n} . \quad(G=\bigcup\{T(\emptyset) \mid T$ is the first component of some condition in $\mathcal{G}\}$.) On this basis we could naively try to define our embedding of $\mathcal{K}$ into the hyperdegrees as follows. For $x \in \mathcal{K} \subseteq \mathcal{L}$ we let $G_{x}: \omega \rightarrow \omega$ be defined by $G_{x}(n)=G(n)(x)$. (As $G(n) \in \Theta_{n} \subseteq \Theta$ it is a map from $\mathcal{L}$ into $\omega$.) The desired image of $x$ would then be $\operatorname{deg}_{h}\left(G_{x}\right)$. Now the order and join properties of usl representations guarantee that this embedding preserves order and join (on all of $\mathcal{L}$ even). If $x \leq y$ then by the order property we can (recursively in the hyperarithmetic representation $\left.\left\langle\Theta_{i}\right\rangle\right)$ calculate $G_{x}(m)$ from $G_{y}(m)$ by finding any $\alpha \in \Theta_{m}$ with $\alpha(y)=G_{y}(m)$ and declaring that $G_{x}(m)=\alpha(x)$. (Such an $\alpha$ exists since $G(m)$ is one.) Similarly if $x \vee y=z$ then, by the join property, we can calculate $G_{z}(m)$ from $G_{x}(m)$ and $G_{y}(m)$ by finding any $\alpha \in \Theta_{m}$ such that $\alpha(x)=G_{x}(m)$ and $\alpha(y)=G_{y}(m)$ and declaring that $G_{z}(m)=\alpha(z)$. (Again $G(m)$ is such an $\alpha$.)

Were congruences modulo $x$ always preserved for every $x$, we could directly carry out the diagonalization and other requirements as well for this definition of $G_{x}$. (Of course, we cannot allow this to happen as it would produce an embedding of all of $\mathcal{L}$ as an initial segment of $\mathcal{D}_{h}$ in place of the one wanted of $\mathcal{K}$.) In actuality, not all congruences are preserved as we refine to various subtrees in our construction. Thus we must modify the definition of the images in $\mathcal{D}_{h}$ and provide nice representations of the hyperdegrees corresponding to $x$. To that end we introduce some dense sets that our generic must meet.

Lemma 2.11. For each $x \in \mathcal{K}$ and $k \in \omega$ the sets $\{P \mid x \in K(P)\}$ and $\{P \mid k(P) \geq k\}$ are dense.

Proof. Consider any $Q \in \mathcal{P}, x \in \mathcal{K}$ and $k \in \omega$. Let $\mathcal{K}^{\prime}$ be the slsl of $\mathcal{K}$ generated by $K(Q)$ and $x$ and let $i \geq k(Q), k$ be such that $\mathcal{K}^{\prime} \subseteq \mathcal{L}_{i}$. Define $S$ with $k(S)=i$ by $S(\sigma)=\operatorname{Tr}(Q)\left(0^{i-k(Q) \wedge} \sigma\right)$. Clearly, $\left\langle S, \mathcal{K}^{\prime}\right\rangle \leq_{\mathcal{P}} Q$ and is in both required sets.

From now on assume that any generic filter $\mathcal{G}$ we consider meets the dense sets of Lemma 2.11. They suffice to define our generic $G$ as above. More crucially, they allow us to define nice representatives of the desired images of $x$ in $\mathcal{D}_{h}$.

Definition 2.12. If $\mathcal{G}$ is a generic filter meeting the dense sets of Lemma 2.11, $G$ the corresponding element of $\prod_{n=0}^{\infty} \Theta_{n}, P \in \mathcal{G}$ and $x \in K(P)$ then $G^{P}$ is the sequence $\left\langle\alpha_{n} \mid n \in \omega\right\rangle$ where $\operatorname{Tr}(P)\left(\left\langle\alpha_{n} \mid n<m\right\rangle\right) \subseteq G$ for every $m$. (Thus $\left\langle\alpha_{n}\right\rangle$ is the path that $G$ follows in the domain of $\operatorname{Tr}(P)$. It is obvious from the definitions that $G$ is a path on (i.e. in the range of) $\operatorname{Tr}(Q)$ for every $Q \in \mathcal{G}$.) We define $G_{x}^{P}(n)$ as $\alpha_{n}(x)$. (In the parlance of Lachlan and Lebeuf [1976], $G^{P}$ is the signature of $G$ on $\operatorname{Tr}(P)$.)

The crucial point is that the hyperdegree of $G_{x}^{P}$ does not depend on $P$. 
Lemma 2.13. If $x \in K(P), K(Q)$ for $P, Q$ in a generic $\mathcal{G}$, then $G_{x}^{P} \equiv_{h} G_{x}^{Q}$.

Proof. As there is an $R \leq P, Q$ in $\mathcal{G}$ by the compatibility of all conditions in a generic filter, it suffices to consider the case that $Q \leq P$. Let $G^{P}=\left\langle\alpha_{n}\right\rangle$ and $G^{Q}=\left\langle\beta_{n}\right\rangle$. By the definition of subtree there is for each $n$ an $m(n)$ such that $\operatorname{Tr}(Q)\left(\left\langle\beta_{s} \mid s<n\right\rangle\right)=$ $\operatorname{Tr}(P)\left(\left\langle\alpha_{m(s)} \mid s<n\right\rangle\right)$ and we can compute the function $m$ recursively in the trees. (By the uniformity of the trees there is for each $n$ a unique $m(n)$ such that $|\operatorname{Tr}(Q)(\sigma)|=$ $|\operatorname{Tr}(P)(\tau)|$ for every $\sigma$ of length $n$ and every $\tau$ of length $m(n)$.) Moreover, by our definition of subtree, $\beta_{n+1}=\alpha_{m(n)+1}$. Thus $G_{x}^{Q}(n)=\beta_{n}(x)=\alpha_{m(n)}(x)=G_{x}^{P}(m(n))$ and so $G_{x}^{Q} \leq_{h} G_{x}^{P}$. The other direction depends on the congruence preservations for $x$ implied by $\operatorname{Tr}(Q) \subseteq K(P) \operatorname{Tr}(P)$.

Suppose that we have, by a hyperarithmetic recursion, determined $G_{x}^{P}(i)=\alpha_{i}(x)$ for $i \leq m(n)$. The next step followed by $G$ in $Q$ is $\beta_{n+1}=\alpha_{m(n)+1}$. It corresponds to the sequence $\left\langle\alpha_{i} \mid m(n)+1 \leq i<m(n+1)\right\rangle$. The definition of $\subseteq_{K(P)}$ implies that $\left\langle\alpha_{i}(x) \mid m(n)+1 \leq i<m(n+1)\right\rangle$ is uniquely determined by $\beta_{n+1}(x)$ to continue the recursion.

Thus given a generic $\mathcal{G}$ we can define a map from $\mathcal{K}$ into $\mathcal{D}_{h}$ by sending $x \in \mathcal{K}$ to $\operatorname{deg}_{h}\left(G_{x}^{P}\right)$ for any $P \in \mathcal{G}$ with $x \in K(P)$. Our naive proofs of the preservation of order an join can now be made precise by simply applying them to $G^{P}$ on $\operatorname{Tr}(P)$ (in place of $G$ on $I d)$ for any $P \in \mathcal{G}$ with $x, y, z \in K(P)$.

Now we must define, analyze and exploit our forcing language and relation to prove that the embedding corresponding to a sufficiently generic $G$ preserves nonorder and produces an initial segment of the hyperdegrees.

We proceed essentially as for (perfect) forcing with binary trees in Sacks [1990, IV.4]. Our base structure (model) is formally the ramified analytic hierarchy up to $\omega_{1}^{C K}$ (ChurchKleene $\omega_{1}$, the first nonrecursive ordinal) which we denote by $\mathcal{M}$. This is the analog of $L_{\omega_{1}^{C K}}$ where the language and definitions are set in second order arithmetic instead of set theory. The sets in the structure are, however, just those subsets of $\omega$ that appear in $L_{\omega_{1}^{C K}}$ or equivalently the hyperarithmetic ones. Our forcing extension will be $\mathcal{M}\left(\omega_{1}^{C K}, G\right)$ which is defined analogously to $L_{\omega_{1}^{C K}}[G]$. If $\omega_{1}^{G}$, the least ordinal not recursive in $G$, is $\omega_{1}^{C K}$ (as will be the case for generic $G$ ) then the sets in $\mathcal{M}\left(\omega_{1}^{C K}, G\right)$ are precisely those hyperarithmetic in $G$. To define and describe the model and forcing relation we have a ramified forcing language as introduced in Feferman [1965] and described in Sacks [1990, III.4] with a term $\mathcal{G}$ for the function $G$ (in place of $\mathcal{T}$ and the set $T$ ) to describe this structure and define the forcing relation. (We are thinking of everything such as $\mathcal{L}, \mathcal{K}, \mathcal{L}_{i}$ and $\Theta_{i}$ as coded in second order arithmetic.) In addition to the usual paraphernalia of second order arithmetic, the language has ranked set variables $X^{\zeta}$ for ordinals $\zeta<\omega_{1}^{C K}$ which range over the sets constructed by level $\zeta$ in $\mathcal{M}\left(\omega_{1}^{C K}, G\right)$. A formula is ranked if all its set variables are ranked. We refer to Sacks [1990, III.4] for the simultaneous recursive definitions of the sets $\mathcal{M}(\zeta, G)$ ), interpretations of terms $\hat{x} \mathcal{H}(x)$ for the set of numbers satisfying the ranked formula $\mathcal{H}$ and for the truth of ranked and unranked formulas in 
the structure $\mathcal{M}\left(\omega_{1}^{C K}, G\right)$. The definitions proceed through inductions on a reasonably defined notion of "full ordinal rank" of a formula. Sacks also provides an analysis of the complexity of the satisfaction relation for ranked (and unranked) formulas. Of course, unranked formulas are interpreted as having their unranked variables ranging over all of $\mathcal{M}\left(\omega_{1}^{C K}, G\right)$. As the change from his setting to ours is purely notational, going from a set (element of $2^{\omega}$ ) to a function in $D$, the development there carries over with only notational changes.

The crucial starting point of the definition of the forcing is that $\langle T, \hat{\mathcal{K}}\rangle \Vdash \mathcal{F}$ for a ranked formula $\mathcal{F}$ if and only if $\mathcal{M}\left(\omega_{1}^{C K}, G\right) \models \mathcal{F}$ for every $G \in[T]$ (the paths through $T)$. The relation is then defined on the unranked formulas by induction on the usual complexity of formulas. The clauses for conjunction and negation are standard: $P \Vdash$ $\mathcal{F} \wedge \mathcal{H} \Leftrightarrow P \Vdash \mathcal{F}$ and $P \Vdash \mathcal{H} ; P \Vdash \neg \mathcal{F} \Leftrightarrow(\forall Q \leq P)(Q \nVdash \mathcal{F})$. There are three cases for the existential quantifiers: $P \Vdash \exists x \mathcal{F}(x) \Leftrightarrow P \Vdash \mathcal{F}(\underline{n})$ for some numeral $\underline{n}$; $P \Vdash \exists X^{\zeta} \mathcal{F}\left(X^{\zeta}\right)$ for $\zeta<\omega_{1}^{C K} \Leftrightarrow P \Vdash \mathcal{F}(\hat{x} \mathcal{H}(x))$ for some $\mathcal{H}$ of rank at most $\zeta ; P \Vdash$ $\exists X \mathcal{F}(X) \Leftrightarrow P \Vdash \exists X^{\zeta} \mathcal{F}\left(X^{\zeta}\right)$ for some $\zeta<\omega_{1}^{C K}$.

The key complexity theoretic fact from Sacks [1990, IV.4] is that the forcing relation for $\Sigma_{1}^{1}$ sentences (those with initial unranked existential set quantifiers followed by a ranked formula) is a $\Pi_{1}^{1}$ relation. This fact is again established quite abstractly and is still true by the same simple argument for our new definition of forcing conditions. The crucial property of perfect forcing in Sacks [1990] that now allows one to prove that for generic $G$ forcing equals truth and $\omega_{1}^{C K}$ is preserved (i.e. $\omega_{1}^{G}=\omega_{1}^{C K}$ ) is the fusion lemma to which we now turn.

\section{The fusion lemma}

We can view the problem of preserving $\omega_{1}^{C K}$ as preserving admissibility in $L_{\omega_{1}^{C K}}$ when we add on the new function $G$. From this point of view, we want to preserve $\Sigma_{1}$ replacement, i.e. $L_{\omega_{1}^{C K}}[G] \models \forall n \in \omega \exists X \varphi(n, X) \rightarrow \exists X \forall n \in \omega\left(\varphi\left(n, X^{[n]}\right)\right.$. Translated into our setting of second order arithmetic and $\mathcal{M}\left(\omega_{1}^{C K}, G\right)$ this is equivalent to preserving $\Delta_{1}^{1}$-comprehension. The key in either case is what is called the fusion lemma. It it is a natural step from the replacement version when one replaces satisfaction by forcing and wants to prove that one can densely decide sentences of the desired form (as is needed to show the usual equivalence between forcing and truth).

Lemma 3.1 (Fusion). Let $P=\langle T, \hat{\mathcal{K}}\rangle$ be a forcing condition and $\exists \bar{X}_{n} \mathcal{F}_{n}\left(\bar{X}_{n}\right)$ a hyperarithmetic sequence of $\Sigma_{1}^{1}$ sentences with their unranked quantifiers displayed such that $\forall n \forall Q \leq P \exists R \leq Q\left[R \Vdash \exists \bar{X}_{n} \mathcal{F}_{n}\left(\bar{X}_{n}\right)\right]$. Then there is a $Q \leq P$, and even one with $k(Q)=k(P)$ and $K(Q)=K(P)$, such that $\forall n\left(Q \Vdash \exists \bar{X}_{n} \mathcal{F}_{n}\left(\bar{X}_{n}\right)\right)$. Moreover, there is a $\zeta<\omega_{1}^{C K}$ such that $\forall n\left(Q \Vdash \exists \bar{X}_{n}^{\zeta} \mathcal{F}_{n}\left(\bar{X}_{n}^{\zeta}\right)\right)$.

Now what goes wrong with an attempted proof of this theorem if we use some standard type of sequential representations $\Theta_{i}$ for $\mathcal{L}_{i}$ and the associated trees? When we try to 
prove the fusion lemma, we thin out our given tree (above each node successively) and get conditions that are trees for $\Theta_{i}$ for larger and larger $i$ as we treat each $\mathcal{F}_{n}$ in turn. As the trees are of different shapes for each $i$ and the representations not nicely nested, there is no way to combine (fuse) the subtrees into the single condition $Q$ required. The first crucial property of our representation is that it allows us to use trees that are essentially (i.e. eventually) of the same shape with splittings for a cofinal segment of the $\Theta_{i}$. We now turn to the basic lemma that conveys the other technical facts about our notion of forcing that enables us to overcome this problem. It was for this application that our lattice representation theorem (Theorem 5.1) was designed.

Proposition 3.2. If $\left\langle T_{1}, \mathcal{K}_{1}\right\rangle \leq_{\mathcal{P}}\left\langle T_{0}, \mathcal{K}_{0}\right\rangle$ then $\left\langle T_{1}, \mathcal{K}_{0}\right\rangle \leq_{\mathcal{P}}\left\langle T_{0}, \mathcal{K}_{0}\right\rangle$. Moreover, we can get an $S$ with $[S] \subseteq\left[T_{1}\right]$ such that $k(S)=k\left(T_{0}\right)$ and $\left\langle S, \mathcal{K}_{0}\right\rangle \leq_{\mathcal{P}}\left\langle T_{0}, \mathcal{K}_{0}\right\rangle$. Thus, if $\mathcal{F}$ is a ranked formula, $P$ is a condition, $Q \leq P$ and $Q \Vdash \mathcal{F}$, then there is an $R \leq P$ such that $R \Vdash \mathcal{F}, k(R)=k(P)$ and $K(R)=K(P)$.

Proof. That $\left\langle T_{1}, \mathcal{K}_{0}\right\rangle \leq_{\mathcal{P}}\left\langle T_{0}, \mathcal{K}_{0}\right\rangle$ is clear from the definition of extension. To get the desired $S$, begin with $T_{1}$. Now $k\left(T_{1}\right)=k_{1} \geq k_{0}=k\left(T_{0}\right)$ and so the branchings at the $n t h$ level of $T_{1}$ which correspond to the elements of $\Theta_{k_{1}+n}$ contain the branchings in $T_{0}$ which correspond to the elements of $\Theta_{k_{0}+n}$ as by our representation theorem (Theorem 5.1), $\Theta_{k_{0}+n} \subseteq \Theta_{k_{1}+n}$. To be more precise we define $S(\emptyset)=T_{1}(\emptyset)$ and $S(\sigma)$ for $\sigma \in$ $\prod_{n=0}^{n=m} \Theta_{k_{0}+n}$ as $T_{1}(\sigma)$. So for every $\sigma$ in its domain $S(\sigma)=T_{1}(\sigma)$. It is then clear that $[S] \subseteq\left[T_{1}\right], k(S)=k\left(T_{0}\right)$ and $\left\langle S, \mathcal{K}_{0}\right\rangle \leq_{\mathcal{P}}\left\langle T_{0}, \mathcal{K}_{0}\right\rangle$ as required. Now if $Q \leq P$ and $Q \Vdash \mathcal{F}$ with $\mathcal{F}$ ranked then this argument gives us an $R \leq P$ with $[R] \subseteq[Q], k(R)=k(P)$ and $K(R)=K(P)$. As $\mathcal{F}$ is ranked and $Q \Vdash \mathcal{F},[R] \subseteq[Q]$ implies that $R \Vdash \mathcal{F}$ as well by the definition of forcing for ranked sentences.

For those familiar with the proof of the fusion lemma in other settings, we note how our special representation allows us to avoid changing the shape of the trees or increasing the slsl $\hat{\mathcal{K}}$ with respect to which we are preserving congruences. The point is that when we have some condition $P=\left\langle T_{0}, \mathcal{K}_{0}\right\rangle$ and ask if there is any $Q=\left\langle T_{1}, \mathcal{K}_{1}\right\rangle$ extending $P$ that forces some ranked formula $\mathcal{F}$ we can restrict ourselves to those with $k\left(T_{1}\right)=k\left(T_{0}\right)$ and $\mathcal{K}_{1}=\mathcal{K}_{0}$ by Proposition 3.2. This idea is really the only new one needed to carry out the proof of the fusion lemma.

Proof of Fusion Lemma. As the forcing relation for $\Sigma_{1}^{1}$ sentences is $\Pi_{1}^{1}$ and extension is arithmetic by definition, the relation $R \leq Q \& k(R)=k(P) \& K(R)=K(P) \& R \Vdash$ $\exists \bar{X}_{n} \mathcal{F}_{n}\left(\bar{X}_{n}\right)$ is $\Pi_{1}^{1}$ and it can be uniformized to a partial $\Pi_{1}^{1}$ function $R(n, Q)$ by Kreisel uniformization (Sacks [1990 II.2.6]). Our hypothesis and Proposition 3.2 together imply that $R$ is defined for every $\langle n, Q\rangle$ with $Q \leq P$. We now define a tree $S$ by recursion so that $\langle S, \hat{\mathcal{K}}\rangle$ is the forcing condition required in the Lemma. In fact, along with the definition of $S(\sigma)$ by induction on the length $n$ of $\sigma$ we define an auxiliary sequence of conditions $\left\langle U_{n, i}, \hat{\mathcal{K}}\right\rangle \leq P$ with $k\left(U_{n, i}\right)=k(P)$ and $\left|U_{n, i}(\emptyset)\right|=|S(\sigma)|$ (which depends only 
on $n$ by uniformity) for $i \leq m(n)$ where $m(n)$ is one less than the number of branches on $T$ at level $n$. Indeed we will have that $\left\langle U_{n, i}^{T(\sigma)}, \hat{\mathcal{K}}\right\rangle \leq P$ for each $\sigma$ of length $n$. (Recall Definition 2.6 and Lemma 2.10.) We begin with $S(\emptyset)=T(\emptyset)$. Suppose we have defined $S(\sigma)$ for all $\sigma$ with length $n$ and have the corresponding $U_{n, m(n)}$. For any $\sigma$ of length $n$ and any appropriate $\alpha$ we let $S\left(\sigma^{\wedge} a\right)=U_{n, m(n)}^{S(\sigma)}\left(0^{n \wedge} \alpha\right)$. Thus $S$ (up to this level) is clearly uniform and a subtree of $T$ that preserves the congruences in $\hat{\mathcal{K}}$. We now define the $U_{n+1, i}$ for $i<m(n+1)$ by induction on $i$. We begin with $\sigma_{0}=\rho^{\wedge} \alpha$ (for some $\rho$ of length $n$ and some $\alpha$ in the appropriate $\Theta_{j}$ ). We let $U_{n+1,0}$ be the first component of $R\left(n+1,\left(U_{n, m(n)}\right)_{0^{n}{ }^{n} \alpha}^{S\left(\sigma_{0}\right)}\right)$. Given $U_{n+1, i}$ and $\sigma_{i+1}$ we let $U_{n+1, i+1}$ be the first component

of $R\left(n+1, U_{n+1, i}^{S\left(\sigma_{i}\right)}\right)$. (So, by induction, $\left\langle U_{n+1, i}, \hat{\mathcal{K}}\right\rangle \leq P$ and $\left\langle U_{n+1, i}, \hat{\mathcal{K}}\right\rangle \Vdash \mathcal{F}_{n+1}$.) This completes the inductive definition of the $U_{n+1, i}$ and so of $S$. The crucial fact about $S$ is that for each $n$ and $\sigma$ of length $n$ every path on $S$ that contains $S(\sigma)$ is a path on $U_{n, i}$ for some $i$ and so makes $\mathcal{F}_{n}$ true by our choice of $U_{n, i}$ as the first component of value of $R(n, U)$ for some $U$ with $\langle U, \hat{\mathcal{K}}\rangle \leq P$. Thus $\mathcal{F}_{n}$ is true of every path on $S$ for every $n$, i.e. $\forall n\left(\langle S, \hat{\mathcal{K}}\rangle \Vdash \mathcal{F}_{n}\right)$.

Given the fusion lemma for our forcing, the remaining development of the basic facts about our forcing is routine and follows Sacks [1990, IV.4]. In particular, essentially the same proofs show first that for every $P$ and $\mathcal{F}$ there is a $Q \leq P$ that decides $\mathcal{F}$, i.e. $Q \Vdash \mathcal{F}$ or $Q \Vdash \neg \mathcal{F}$ and then that for any generic filter $\mathcal{G}$ (i.e. one such that for every $\mathcal{F}$ there is a $P \in \mathcal{G}$ that decides $\mathcal{F}$ ) forcing equals truth in the sense that for, every $\mathcal{F}$, $\mathcal{M}\left(\omega_{1}^{C K}, G\right) \models \mathcal{F} \Leftrightarrow(\exists P \in \mathcal{G})(P \Vdash \mathcal{F})$.

Remark 3.3. One can simplify Sacks's inductive proof that forcing equals truth by constructing a generic sequence $P_{i}$ of conditions such that each sentence $\mathcal{F}$ of our language is decided by some $P_{i}$ with the sentences arranged so that both $\mathcal{F}$ and $\mathcal{H}$ are decided before deciding $\mathcal{F} \& \mathcal{H}$. This would allow one to avoid having to prove an analog of Sacks's Proposition IV.4.6 as the case of a conjunction in Sacks's inductive proof that forcing equals truth is now immediate.

In addition to meeting the dense sets of conditions deciding each $\mathcal{F}$, we explicitly meet certain other sets of conditions to assure that we can define our embedding on all of $\mathcal{K}$ and show that it is a one-one map onto the hyperdegrees below the generic $G=G_{1}^{I d}$.

\section{Initial segment verifications}

To simplify our notation for the sets hyperarithmetic in $G$, i.e. those in $\mathcal{M}\left(\omega_{1}^{C K}, G\right)$, and to emphasize the analogy to the Turing degree constructions of initial segments, we number the terms $\hat{x} \mathcal{H}$ of our language by ordinals $\delta<\omega_{1}^{C K}$ and denote the characteristic function of the set they stand for by $\{\delta\}^{G}$. (Note that, in contrast to the situation with Turing reductions, these are all total functions but, of course, the list is only recursive in $\mathcal{O}$.) We at times use $\delta_{x}^{P}$ to name the reduction such that $\left\{\delta_{x}^{P}\right\}^{G}=\{\delta\}^{G_{x}^{P}}$. 
To assure that our embedding preserves nonorder we want to show for any $x \not \leq y$ in $\mathcal{K}$, condition $P$ with $x, y \in K(P)$ and $\delta<\omega_{1}^{C K}$, that $\left\{Q \mid Q \Vdash\left\{\delta_{y}^{P}\right\}^{G} \neq G_{x}^{P}\right\}$ is dense below $P$. In addition we want to show that $\left\{Q \mid Q \Vdash \exists x \in \mathcal{K}\left(Q \Vdash\{\delta\}^{G} \equiv_{h} G_{x}^{P}\right)\right\}$ is also dense below any $P$ for each $\delta$. These two results would then finish the proof or our theorem. We begin with an auxiliary collection of dense sets that make our task much simpler. They correspond to the total subtrees of Lerman [1983] and allow us to convert the arguments for $\mathcal{D}_{T}$ to our setting of $\mathcal{D}_{h}$.

Lemma 4.1 (Totality). For each condition $P$ and $\delta<\omega_{1}^{C K}$ there is a $Q \leq P$ with $k(Q)=k(P)$ and $K(Q)=K(P)$ such that for every $x$ and $\sigma$ of length $x$ there is an $n \in\{0,1\}$ for which $Q_{\sigma} \Vdash\{\delta\}^{G}(x)=\underline{n}$. Moreover, the map $q$ taking $\langle x, \sigma\rangle$ to this $n$ is hyperarithmetic.

Proof. For any $Q \leq P$ and any $m$ there is clearly an $R \leq Q$ and an $n \in\{0,1\}$ for which $R \Vdash\{\delta\}^{G}=\underline{n}$. (Otherwise by the density of deciding formulas we could find $R_{1} \leq R_{0} \leq Q$ such that $R_{n} \Vdash\{\delta\}^{\mathcal{G}}(\underline{m}) \neq \underline{n}$ (for $n=0,1$ ) contradicting one of the basic facts about forcing.) Now applying the construction of the fusion lemma, with the $\Pi_{1}^{1}$ operator taking $m, Q$ to such an $R$ (and $n$ ) for the nodes at level $m$, gives the desired condition which, as usual, has by construction the same $k$ and $K$ values as $P$.

Proposition 4.2 (Diagonalization). For any $x \not \leq y$ in $\mathcal{K}, \delta<\omega_{1}^{C K}$ and $P$ in our generic filter with $x, y \in K(P)$ there is a $Q \leq P$ such that $Q \Vdash\left\{\delta_{y}^{P}\right\}^{G} \neq G_{x}^{P}$.

Proof. Begin by taking $Q \leq P$ as in Lemma 4.1 for $\delta_{y}^{P}$. We then choose any $\alpha_{0}, \alpha_{1} \in \Theta_{k(P)}$ such that $\alpha_{0} \equiv_{y} \alpha_{1}$ but $\alpha_{0} \not_{x} \alpha_{1}$. Such $\alpha_{0}$ and $\alpha_{1}$ exist by the differentiation property of usl representations. Suppose $\operatorname{Tr}(Q)(\emptyset)=\operatorname{Tr}(P)(\sigma)$ and so $\operatorname{Tr}(Q)\left(\alpha_{i}\right) \supseteq \operatorname{Tr}(P)\left(\sigma^{\wedge} \alpha_{i}\right)$ for $i \in\{0,1\}$. Now clearly any condition $Q_{i} \leq Q_{\alpha_{i}}$ forces $G_{x}^{P}(|\sigma|)=\alpha_{i}(x)$. Let $\beta_{i}=\left(\alpha_{i}\right)^{|\sigma|}$, i.e. the concatenation of $|\sigma|$ many copies of $\alpha_{i}$. Consider then the conditions $Q_{\beta_{i}}$. They still force $G_{x}^{P}(|\sigma|)=\alpha_{i}(x)$. On the other hand by Lemma 4.1 they each force a value for $\left\{\delta_{y}^{P}\right\}^{G}$ at $|\sigma|$.

As the $\beta_{i}$, for $i=0,1$, are congruent modulo $y$ and $y \in K(P)$, the initial segments of $G_{y}^{P}$ that $Q_{\beta_{i}}(\emptyset)$ determine are congruent modulo $y$ as well. If $G$ is any path through $Q_{\beta_{0}}$ then (by uniformity of $\operatorname{Tr}(Q)$ ) changing its initial segment $Q_{\beta_{0}}(\emptyset)$ to $Q_{\beta_{1}}(\emptyset)$ produces a path $G^{\prime}$ through $Q_{\beta_{1}}$ and so a corresponding one through $\operatorname{Tr}(P)$ that is congruent to $G^{P}$ modulo $y$. As the value of $\{\delta\}^{G_{y}^{P}}=\left\{\delta_{y}^{P}\right\}^{G}$ is determined by $G_{y}^{P}$ the two conditions must force the same value for $\{\delta\}^{G_{y}^{P}}(|\sigma|)$. This value must be different from one of $\alpha_{i}(x)$ $=0,1$. Thus one of the $Q_{\beta_{i}}$ forces $\{\delta\}^{G_{y}^{P}}=\left\{\delta_{y}^{P}\right\}^{G} \neq G_{x}^{P}$ as required.

We turn now to the requirement that the image of $\mathcal{K}$ under our embedding form an initial segment of $\mathcal{D}_{h}$. This argument is somewhat more complicated than those above and uses both the meet and homogeneity interpolants. Still, given Lemma 4.1, we present an argument that would work for $\mathcal{D}_{T}$ as well. In that setting it would be simpler 
than the existing proofs in the literature because of our changes to the definition of the homogeneity interpolants.

We begin with the notion of a $\delta$-splitting and a lemma about such splittings.

Definition 4.3. Given a $P=\langle T, \hat{\mathcal{K}}\rangle$ in our generic filter with $w \in \hat{\mathcal{K}}$, a reduction $\delta$, and a condition $Q \leq P$ and function $q$ both as in Proposition 4.1, we say that $\sigma$ and $\tau$ (of the same length) are a $\delta$-splitting (or $\delta$-split) on $Q$ (modulo $w$ ) if ( $\sigma \equiv_{w} \tau$ and) there is an $n \leq|\sigma|$ such that $q\left(n, \sigma\lceil n) \neq q(n, \tau \mid n)\right.$. (So for any paths $G_{0}$ and $G_{1}$ on $Q$ extending $\operatorname{Tr}(Q)(\sigma)$ and $\operatorname{Tr}(Q)(\tau)$, respectively, $\{\delta\}^{G_{0}}(n) \neq\{\delta\}^{G_{1}}(n)$.) If $R \leq Q, R(\mu)=Q(\sigma), R(\nu)=Q(\tau)$ and $\sigma$ and $\tau \delta$-split (modulo $w$ ) on $Q$ then we also say that $\mu$ and $\nu \delta$-split on $R$ (modulo $w$ ).

Lemma 4.4. Given $P$ and $Q$ as in Lemma 4.1, there is a $\rho$ such that the set $S p(\rho)=$ $\left\{w \in K(P) \mid\right.$ there are no $\sigma, \tau$ that $\delta$-split on $\operatorname{Tr}(Q)_{\rho}$ modulo $\left.w\right\}$ is maximal. Moreover, this maximal set is closed under meet and so has a least element $z$.

Proof. Let $T=\operatorname{Tr}(Q)$ and $k=k(Q)=k(P)$ and $\hat{\mathcal{K}}=K(P)=K(Q)$. As $\hat{\mathcal{K}}$ is finite there is clearly a $\rho$ such that $S p(\rho)$ is maximal. Note that then $S p(\mu)=S p(\rho)$ for any $\mu \supseteq \rho$ as $\operatorname{Tr}(Q)_{\mu} \subseteq_{\hat{\mathcal{K}}} \operatorname{Tr}(Q)_{\rho}$. Consider any $x, y \in S p(\rho)$ with $x \wedge y=w$. As $\hat{\mathcal{K}} \subseteq_{l s l} \mathcal{L}, w \in \hat{\mathcal{K}}$. To show that $S p(\rho)$ is closed under meet it suffices (by the maximality of $S p(\rho)$ ) to show that there is no $\delta$-splitting on $Q_{\rho^{\wedge} 0}$ modulo $z$. Let $\hat{k}$ denote $k\left(Q_{\rho^{\wedge} 0}\right)=k(Q)+|\rho|+1$. Suppose there were such a split $\bar{\mu}$ and $\bar{\nu}$. By our definition of $Q_{\rho^{\wedge} 0}, \bar{\mu}, \bar{\nu} \in \prod_{n=0}^{n=m} \Theta_{\hat{k}+n}$ for some $m$. The splitting in $Q_{\rho}$ at the corresponding levels, however, have branchings for all elements of $\Theta_{\hat{k}+n+1}$. Thus, by the existence of meet interpolants for $\Theta_{\hat{k}+n}$ in $\Theta_{\hat{k}+n+1}$, there are $\bar{\gamma}_{0}, \bar{\gamma}_{1}, \bar{\gamma}_{2} \in \prod_{n=0}^{n=m} \Theta_{\hat{k}+n+1}$ such that for each $j \leq m$, the $\bar{\gamma}_{i}(j)$ for $i \in\{0,1,2\}$ are meet interpolants for $\bar{\mu}(j)$ and $\bar{\nu}(j)$, i.e. $\bar{\mu} \equiv_{x} \bar{\gamma}_{0} \equiv_{y} \bar{\gamma}_{1} \equiv_{x} \bar{\gamma}_{2} \equiv_{x} \bar{\nu}$. As $\bar{\mu}$ and $\bar{\nu}$ form a $\delta$-splitting on $Q_{\rho^{\wedge} 0}$ so do one of the successive pairs such as $\bar{\gamma}_{0}, \bar{\gamma}_{1}$. But then $0^{\wedge} \bar{\gamma}_{0}$ and $0^{\wedge} \bar{\gamma}_{1}$ would be a $\delta$-split on $Q_{\rho}$ congruent modulo $y$ for a contradiction. (The situations for the other pairs are the same but perhaps with $x$ in place of $y$.)

We now build the analog of what is often called a $\delta$-splitting tree in the Turing degree setting. It is in the construction of these trees that our new definition of homogeneity interpolants simplifies the construction as compared, for example, to that of Lerman [1983, VII.3].

Proposition 4.5 (Splitting trees). Given $\delta, z, P, Q$ and $\rho$ as in Lemma 4.4, there is a condition $\langle S, \hat{\mathcal{K}}\rangle \leq Q_{\rho}$ (with $k(S)=k(Q)=k(P)=k$ ) such that for any $\sigma \in$ dom $S(=$ $\operatorname{dom} Q=\operatorname{dom} P$ ) and any $\alpha, \beta$ in the appropriate $\Theta_{j}$ (actually $j=k+|\sigma|$ ), if $\alpha \neq_{z} \beta$ then $\sigma^{\wedge} \alpha$ and $\sigma^{\wedge} \beta \delta$-split on $S$.

Proof. We define $S(\sigma)$ (with $k(S)=k$ ) by induction on $|\sigma|$ beginning, of course, with $S(\emptyset)=Q_{\rho}(\emptyset)$. Suppose we have defined $S(\sigma)=Q\left(\tau_{\sigma}\right)$ for all $\sigma$ of length $n$. We must 
define $S\left(\sigma^{\wedge} \alpha\right)$ for all such $\sigma$ and appropriate $\alpha$ as extensions $Q\left(\tau_{\sigma^{\wedge} \alpha}\right)$ of $Q\left(\tau_{\sigma^{\wedge}}{ }^{\wedge}\right)$ obeying all the congruences in $\hat{\mathcal{K}}$, i.e. if $x \in \hat{\mathcal{K}}$ and $\alpha \equiv_{x} \beta$ then $\tau_{\sigma^{\wedge} \alpha} \equiv_{x} \tau_{\sigma^{\wedge} \beta}$. We list the $\sigma$ of length $n+1$ as $\sigma_{i}^{\wedge} \alpha_{i}$ for $i<m=\left|\prod_{j=0}^{j=n} \Theta_{k+j}\right|$ and define by induction on $r$ strings $\rho_{i, r}$ for $i<m, r<l=m(m+1) / 2$ (the number of pairs $\{i, j\}$ with $i, j<m$ ). At the end of our induction we will set $\tau_{\sigma_{i}{ }^{\wedge} \alpha_{i}}=\tau_{\sigma_{i}}{ }^{\wedge} \rho_{i, 0}{ }^{\wedge} \ldots{ }^{\wedge} \rho_{i, l-1}$. For this to succeed it suffices to guarantee for every $i, j<m$ and $w \in \hat{\mathcal{K}}$ that $\alpha_{i} \equiv_{w} \alpha_{j} \Rightarrow \rho_{i, r} \equiv_{w} \rho_{j, r}$ for every $r<l$ and that if $\alpha_{i} \not \equiv_{z} \alpha_{j}$ then $\tau_{\sigma_{i}}{ }^{\wedge} \rho_{i, 0}{ }^{\wedge} \ldots \wedge \rho_{i, r}$ and $\tau_{\sigma_{j}}{ }^{\wedge} \rho_{j, 0}{ }^{\wedge} \ldots{ }^{\wedge} \rho_{r} \delta$-split on $Q$ where $r<l$ is (the code for) $\{i, j\}$.

By induction on $r<l$ we suppose we have $\tau_{\sigma_{i}}{ }^{\wedge} \rho_{i, 0}{ }^{\wedge} \ldots{ }^{\wedge} \rho_{i, r-1}=\nu_{i}$ for all $i<m$ and that $\{p, q\}$ is pair number $r$. If $\alpha_{p} \equiv_{z} \alpha_{q}$ there is no requirement to satisfy and we let $\rho_{i, r}=\emptyset$ for every $i$. Otherwise, let $w$ be the largest $y \in \mathcal{L}_{k+n}$ such that $\alpha_{p} \equiv_{y} \alpha_{q}$. (To see that there is a largest such $y$, first note that $\mathcal{L}_{k+n}$ is a lattice as it is a finite lsl. As $\Theta_{k+n}$ is an usl representation for $\mathcal{L}_{k+n}$, if $\alpha_{p} \equiv_{u, v} \alpha_{q}$ for $u, v \in \mathcal{L}_{k+n}$ then $\alpha_{p} \equiv_{t} \alpha_{q}$ where $t$ is the least element of $\mathcal{L}_{k+n}$ above both $u$ and $v$ (their join from the viewpoint of $\mathcal{L}_{k+n}$ ). Thus, there is a largest $y$ as desired.) Of course, $z \not \leq w$. By our choice of $z$ there are $\sigma, \tau \in \prod_{n=0}^{n=s} \Theta_{k+n}$ such that $\nu_{p}$ extended by $\sigma$ and $\tau$ form a $\delta$-splitting congruent modulo $w$. (We can find such a split on $Q_{\nu_{p}}$ by definition of $\rho$ and $z$ and our assumption on $w$. It translates into such $\sigma$ and $\tau$.) Consider $\nu_{q}{ }^{\wedge} \tau$. It must form a $\delta$-splitting on $Q$ with one of $\nu_{p}{ }^{\wedge} \sigma$ and $\nu_{p}{ }^{\wedge} \tau$ by the basic properties of $Q$. If it splits with the latter string then we can set $\rho_{i, r+1}=\tau$ and clearly fulfill the requirements for this pair $\{p, q\}$ both for congruence modulo $w$ (as all new extensions are identical) and $\delta$-splitting. Thus we assume that $\nu_{p}{ }^{\wedge} \sigma$ and $\nu_{q}{ }^{\wedge} \tau \delta$-split on $Q$. We now use our homogeneity interpolants.

We know that $w$ is the largest $y \in \mathcal{L}_{n+k}$ such that $\alpha_{p} \equiv_{y} \alpha_{q}$ and that $\sigma \equiv_{w} \tau$. Thus for any $z \in \hat{\mathcal{K}} \subseteq \mathcal{L}_{k+n}$ if $\alpha_{p} \equiv_{z} \alpha_{q}$ then $z \leq w$ and so $\sigma \equiv_{z} \tau$. By Theorem 5.1(3) we can now find homogeneity interpolants $\gamma_{0}(s), \gamma_{1}(s)$ in $\Theta_{k+s+1}$ and associated $\hat{\mathcal{K}}$-homomorphisms $f_{s}: \alpha_{p}, \alpha_{q} \mapsto \sigma(s), \gamma_{1}(s), g_{s}: \alpha_{p}, \alpha_{q} \mapsto \gamma_{0}(s), \gamma_{1}(s)$ and $h_{s}: \alpha_{p}, \alpha_{q} \mapsto \gamma_{0}(s), \tau(s)$ for each $s<|\sigma|=|\tau|$. (We let $\alpha_{0}=\alpha_{p}, \alpha_{1}=\alpha_{q}, \beta_{0}=\sigma(s), \beta_{1}=\tau(s), \hat{\mathcal{L}}=\hat{\mathcal{K}}$ and $i=k+n$ in the Theorem.) As $\nu_{p}{ }^{\wedge} \sigma$ and $\nu_{q}{ }^{\wedge} \tau \delta$-split on $Q$ one of the pairs $\nu_{p}{ }^{\wedge} \sigma, \nu_{q}{ }^{\wedge} \bar{\gamma}_{1} ; \nu_{p}{ }^{\wedge} \bar{\gamma}_{0}, \nu_{q}{ }^{\wedge} \bar{\gamma}_{1}$ and $\nu_{p}{ }^{\wedge} \bar{\gamma}_{0}, \nu_{q}{ }^{\wedge} \tau$ must also $\delta$-split on $Q$. Suppose for the sake of definiteness it is the second pair $\nu_{p}{ }^{\wedge} \bar{\gamma}_{0}, \nu_{q}{ }^{\wedge} \bar{\gamma}_{1}$. In this case we let $\rho_{i, r+1}(s)=g_{s}\left(\alpha_{i}\right)$ for every $i$ and $s$. We use $f_{s}$ or $h_{s}$ in place of $g_{s}$ if the $\delta$-splitting pairs are $\nu_{p}{ }^{\wedge} \sigma, \nu_{q}{ }^{\wedge} \bar{\gamma}_{1}$ or $\nu_{p}{ }^{\wedge} \bar{\gamma}_{0}, \nu_{q}{ }^{\wedge} \tau$, respectively. By the homomorphism properties of the interpolants these extensions preserve all the congruences in $\hat{\mathcal{K}}$ between any $\alpha_{i}$ and $\alpha_{j}$ as required to complete the induction and our construction of $\delta$-splitting trees .

We now conclude the proof that $\left\{Q \mid Q \Vdash \exists x \in \mathcal{K}\left(Q \Vdash\{\delta\}^{G} \equiv_{h} G_{x}^{Q}\right)\right\}$ is dense below $P$ for each $\delta$ and $P \in \mathcal{G}$. This will show that our embedding maps onto an initial segment of $\mathcal{D}_{h}$ by proving that the $\delta$-splitting tree $S$ forces that $\{\delta\}^{G} \equiv_{h} G_{z}^{S}$ (for the $z$ of the Lemma). The required fact is generally called the Computation Lemma. We supply the standard proof. 
Lemma 4.6 (Computation Lemma). Given $\delta, z, P, Q, \rho$ and $S$ as in Lemma 4.5, $S \Vdash$ $\{\delta\}^{G} \equiv{ }_{h} G_{z}^{S}$.

Proof. Let $G \in[S]$. We first show that $\{\delta\}^{G} \leq_{h} G_{z}^{S}$. Consider any $n$. Using $G_{z}^{S}$ we can find all the $\sigma \in \operatorname{dom} S$ of length $n$ such that $\sigma(l)=G_{z}^{S}(l)$ for every $l \leq n$. All of these $\sigma$ are congruent modulo $z$ and so all $S_{\sigma}$ force the same value for $\{\delta\}^{G}$ at $n$. As $S(\sigma)$ is an initial segment of $G$ for one of these $\sigma$, this value must be $\{\delta\}^{G}(n)$. We next argue that $G_{z}^{S} \leq_{h}\{\delta\}^{G}$. Consider all $\sigma, \tau \in \operatorname{dom} S$ of length $n$. If $\sigma \not_{z} \tau$ then, by the construction of $S, S_{\sigma}$ and $S_{\tau}$ force different values for $\{\delta\}^{G}$ at some $l<n$. Thus using $\{\delta\}^{G}\lceil n$ we can find the unique congruence class modulo $z$ consisting of those $\sigma$ such that $S(\sigma)$ is not ruled out as a possible initial segment of $G$. For one $\sigma$ in this class, $S(\sigma)$ is an initial segment of $G$ and as all the $\sigma$ in this class are congruent modulo $z$, they all determine the same values of $G_{z}^{S} \uparrow n$ which must then be the correct value.

We have now completed the proof that any generic filter $\mathcal{G}$ (deciding all sentences and meeting the dense sets provided by Lemma 4.1 and Propositions 4.2 and 4.5) provides an embedding of $\mathcal{K}$ onto an initial segment of $\mathcal{D}_{h}$ that sends $x \in \mathcal{K}$ to $\operatorname{deg}_{h}\left(G_{x}^{P}\right.$ ) (for any $P \in \mathcal{G})$.

Forcing ranked sentences is a $\Pi_{1}^{1}$ relation (and so recursive in $\mathcal{O}$ ) and the inductive definition thereafter proceeds by the standard definition quantifying over previous levels of the forcing relation. (Remember that existential quantification is reduced to quantification over ordinals and terms below $\omega_{1}^{C K}$ and so to quantification over $\mathcal{O}$, while negation requires quantification over forcing conditions and so over $\mathcal{O} \oplus \mathcal{K}$.) Thus the forcing relation for arbitrary sentences is arithmetic in $\mathcal{O} \oplus \mathcal{K}$ (at a level simply related to the complexity of the sentence). Thus one can, as usual, construct a generic sequence and corresponding filter that decides all sentences hyperarithmetically in $\mathcal{O} \oplus \mathcal{K}$. The steps for meeting the requirements of totality, diagonalization and splitting are also obviously computable in the forcing relation once one has proven the existence of the desired extensions (Lemma 4.1, Proposition 4.2 and 4.5). Thus we can find our generic $G \equiv_{h} G_{1}^{I d}$ (remember, 1 is the top element of our lattice), such that the hyperdegrees below that of $G$ are isomorphic to our given $\mathcal{K}$, hyperarithmetically in $\mathcal{O} \oplus \mathcal{K}$. This establishes Theorem 1.2 given our lattice representation theorem to whose proof we now turn.

\section{The lattice representation theorem}

Theorem 5.1. If $\mathcal{L}$ is a countable lattice then there is an usl representation $\Theta$ of $\mathcal{L}$ along with a nested sequence of finite slsls $\mathcal{L}_{i}$ starting with $\mathcal{L}_{0}=\{0,1\}$ with union $\mathcal{L}$ and a nested sequence of finite subsets $\Theta_{i}$ with union $\Theta$ with both sequences recursive in $\mathcal{L}$ with the following properties:

1. For each $i, \Theta_{i} \uparrow \mathcal{L}_{i}$ is an usl representation of $\mathcal{L}_{i}$. 
2. There are meet interpolants for $\Theta_{i}$ in $\Theta_{i+1}$, i.e. if $\alpha \equiv_{z} \beta, x \wedge y=z\left(\right.$ in $\Theta_{i}$ and $\mathcal{L}_{i}$, respectively) then there are $\gamma_{0}, \gamma_{1}, \gamma_{2} \in \Theta_{i+1}$ such that $\alpha \equiv_{x} \gamma_{0} \equiv_{y} \gamma_{1} \equiv_{x} \gamma_{2} \equiv_{y} \beta$.

3. For every $\hat{\mathcal{L}} \subseteq_{l s l} \mathcal{L}_{i}$ there are homogeneity interpolants for $\Theta_{i}$ with respect to $\hat{\mathcal{L}}$ in $\Theta_{i+1}$, i.e. for every $\alpha_{0}, \alpha_{1}, \beta_{0}, \beta_{1} \in \Theta_{i}$ such that $\forall w \in \hat{\mathcal{L}}\left(\alpha_{0} \equiv_{w} \alpha_{1} \rightarrow \beta_{0} \equiv_{w} \beta_{1}\right)$, there are $\gamma_{0}, \gamma_{1} \in \Theta_{i+1}$ and $\hat{\mathcal{L}}$-homomorphisms $f, g, h: \Theta_{i} \rightarrow \Theta_{i+1}$ such that $f$ : $\alpha_{0}, \alpha_{1} \mapsto \beta_{0}, \gamma_{1}, g: \alpha_{0}, \alpha_{1} \mapsto \gamma_{0}, \gamma_{1}$ and $h: \alpha_{0}, \alpha_{1} \mapsto \gamma_{0}, \beta_{1}$.

Proof. We first define the sequence $\mathcal{L}_{i}$ of slsls of $\mathcal{L}$ beginning with $\mathcal{L}_{0}$ which consists of the 0 and 1 of $\mathcal{L}$. We let the other elements of $\mathcal{L}$ be $x_{n}$ for $n \geq 1$ and $\mathcal{L}_{n}$ be the (necessarily finite) slsl of $\mathcal{L}$ generated by $\left\{0,1, x_{1}, \ldots, x_{n}\right\}$. As for $\Theta$, we choose a countable set $\alpha_{i}$ and stipulate that $\Theta=\left\{\alpha_{i} \mid i \in \omega\right\}$. We begin defining the (values of) the $\alpha_{i}$ by setting $\alpha_{0}(x)=0$ for all $x \in \mathcal{L}$ and $\alpha(0)=0$ for all $\alpha \in \Theta$. We will now define $\Theta_{n}$ and the values of $\alpha \in \Theta_{n}$ (other than $\alpha_{0}$ ) on the elements of $\mathcal{L}_{n}$ (other than $0)$ by recursion. For $\Theta_{0}$ we choose a new element $\beta$ of $\Theta$ and let $\Theta_{0}=\left\{\alpha_{0}, \beta\right\}$ and set $\beta(1)=1$. Given $\Theta_{n}$ and the values for its elements on $\mathcal{L}_{n}$ we wish to enlarge $\Theta_{n}$ to $\Theta_{n+1}$ and define the values of $\alpha(x)$ for $\alpha \in \Theta_{n+1}$ and $x \in \mathcal{L}_{n+1}$ so that the requirements of the Theorem are satisfied. To do this we prove a number of general extension theorems for usl representations in the Propositions below that show that we can make simple extensions to satisfy any particular meet or homogeneity requirement and also extend usl representations from smaller to larger slsls of $\mathcal{L}$. To be more specific, we first apply Proposition 5.5 successively for each choice of $x \wedge y=z$ in $\mathcal{L}_{n}$ and $\alpha, \beta \in \Theta_{n}$ with $\alpha \equiv_{z} \beta$ choosing new elements of $\Theta$ to form $\Theta_{n}^{\prime}$ extending $\Theta_{n}$ and defining them on $\mathcal{L}_{n}$ so that $\Theta_{n}^{\prime} \uparrow \mathcal{L}_{n}$ is an usl representation for $\mathcal{L}_{n}$ containing $\Theta_{n}$ and the required meet interpolants for every such $x, y, z, \alpha$ and $\beta$. We then apply Proposition 5.6 successively for each $\hat{\mathcal{L}} \subseteq_{l s l} \mathcal{L}_{n}$ and each $\alpha_{0}, \alpha_{1}, \beta_{0}, \beta_{1} \in \Theta_{n}$ such that $\forall w \in \hat{\mathcal{L}}\left(\alpha_{0} \equiv_{w} \alpha_{1} \rightarrow \beta_{0} \equiv_{w} \beta_{1}\right)$ to get larger subset $\Theta_{n}^{\prime \prime}$ of $\Theta$ which we also define on $\mathcal{L}_{n}$ so as to have an usl representation $\Theta_{n}^{\prime \prime} \uparrow \mathcal{L}_{n}$ for $\mathcal{L}_{n}$ that has the required homogeneity interpolants and $\hat{\mathcal{L}}$-homomorphisms from $\Theta_{n}$ into $\Theta_{n}^{\prime \prime}$ for every such $\alpha_{0}, \alpha_{1}, \beta_{0}, \beta_{1} \in \Theta_{n}$. Finally, we apply Proposition 5.4 to define the elements of $\Theta_{n}^{\prime \prime}$ on $\mathcal{L}_{n+1}$ and further enlarge it to our desired finite $\Theta_{n+1} \subseteq \Theta$ with all its new elements also defined on $\mathcal{L}_{n+1}$ so as to have an usl representation of $\mathcal{L}_{n+1}$ with all the properties required by the Theorem. It is now easy immediate from the definitions that the union $\Theta$ of the $\Theta_{n}$ is an usl representation of $\mathcal{L}$.

Notation 5.2. If a finite $\hat{\mathcal{L}}$ is a slsl of $\mathcal{L}, \hat{\mathcal{L}} \subseteq_{l s l} \mathcal{L}$, and $x \in \mathcal{L}$ then we let $\hat{x}$ denote the least element of $\hat{\mathcal{L}}$ above $x$. The desired element of $\hat{\mathcal{L}}$ exists because $\hat{\mathcal{L}}$ is a slsl of $\mathcal{L}$ and so the infimum (in $\hat{\mathcal{L}}$ or, equivalently, in $\mathcal{L}$ ) of $\{u \in \hat{\mathcal{L}} \mid x \leq u\}$ is in $\hat{\mathcal{L}}$ and is the desired $\hat{x}$. As $\hat{\mathcal{L}}$ is finite it is also a lattice but join in $\hat{\mathcal{L}}$ may not agree with that in $\mathcal{L}$. We denote them by $\vee_{\hat{\mathcal{L}}}$ and $\vee_{\mathcal{L}}$ respectively when it is necessary to make this distinction.

Lemma 5.3. With the notation as above, $\hat{x}=x$ for $x \in \hat{\mathcal{L}}$ and so it is an idempotent operation. If $x \leq y$ are in $\mathcal{L}$ then $\hat{x} \leq \hat{y}$. If $x \vee_{\mathcal{L}} y=z$ are in $\mathcal{L}$ then $\hat{z}=\hat{x} \vee_{\hat{\mathcal{L}}} \hat{y}$. 
Proof. The first two assertions follow immediately from the definition of $\hat{x}$. The third is only slightly less immediate: $x, y \leq x \vee_{\mathcal{L}} y=z$ and so by the second assertion, $\hat{x}, \hat{y} \leq \hat{z}$ and so $\hat{x} \vee_{\hat{\mathcal{L}}} \hat{y} \leq \hat{z}$. For the other direction, note that as $x \leq \hat{x}, y \leq \hat{y}$, we have that $z=x \vee_{\mathcal{L}} y \leq \hat{x} \vee_{\mathcal{L}} \hat{y} \leq \hat{x} \vee_{\hat{\mathcal{L}}} \hat{y} \in \hat{\mathcal{L}}$ and so $\hat{z} \leq \hat{x} \vee_{\hat{\mathcal{L}}} \hat{y}$.

Proposition 5.4. If $\Theta$ is a finite usl representation for $\hat{\mathcal{L}} \subseteq_{l s l} \mathcal{L}$ (finite) then there are extensions for each $\alpha \in \Theta$ to maps with domain $\mathcal{L}$ and finitely many further functions $\beta$ with domain $\mathcal{L}$ such that adding them on to our extensions of the $\alpha \in \Theta$ provides an usl representation $\Theta^{\prime}$ of $\mathcal{L}$ with $\Theta \subseteq \Theta^{\prime} \uparrow \hat{\mathcal{L}}$. Moreover, these extensions can be found uniformly recursively in the given data $(\Theta, \hat{\mathcal{L}}$ and $\mathcal{L})$.

Proof. For $\alpha \in \Theta$ and $x \in \mathcal{L}$ set $\alpha(x)=\alpha(\hat{x})$. We first check that we have maintained the order and join properties required of an usl representation. If $x \leq y$ are in $\mathcal{L}, \alpha, \beta \in \Theta$ and $\alpha \equiv_{y} \beta$ then by definition $\alpha \equiv_{\hat{y}} \beta$ and so $\alpha \equiv_{\hat{x}} \beta$ as $\hat{x} \leq \hat{y}$ by Lemma 5.3 and $\Theta$ 's being an usl representation of $\hat{\mathcal{L}}$. Thus, by definition, $\alpha \equiv_{x} \beta$ as required.

Next, if $x \vee_{\mathcal{L}} y=z$ are in $\mathcal{L}$ and $\alpha \equiv_{x, y} \beta$ we wish to show that $\alpha \equiv_{z} \beta$. Again by definition $\alpha \equiv_{\hat{x}, \hat{y}} \beta$. By Lemma 5.3, $\hat{x} \vee_{\mathcal{L}} \hat{y}=z$, so by $\Theta$ being an usl representation for $\hat{\mathcal{L}}, \alpha \equiv_{\hat{z}} \beta$ and so by definition, $\alpha \equiv_{z} \beta$.

All that remains is to show that we can add on new maps with domain $\mathcal{L}$ that provide witnesses for the differentiation property for elements of $\mathcal{L}-\hat{\mathcal{L}}$ while preserving the order and join properties. This is a standard construction. For each pair $x \not \leq y$ (in $\mathcal{L}$ but not both in $\hat{\mathcal{L}}$ ) in turn we add on new elements $\alpha_{x, y}$ and $\beta_{x, y}$ with all new and distinct values at each $z \in \mathcal{L}$ except that they agree on all $z \leq x$ (and at 0 , of course, have value 0 ). These new elements obviously provide the witnesses required for the differentiation property for an usl representation. It is easy to see that they also cause no damage to the order or join properties. There are no new nontrivial instances of congruences between them and the old ones in $\Theta$ (extended to $\mathcal{L}$ ). Among the new elements the only instances to consider are ones between $\alpha_{x, y}$ and $\beta_{x, y}$ for the same pair $x, y$ and for lattice elements $z$ less than or equal to $x$. As $\alpha_{x, y} \equiv_{z} \beta_{x, y}$ for all $z \leq x$, the order and join properties are immediate.

Proposition 5.5. If $\alpha, \beta \in \Theta$, an usl representation for a finite lattice $\mathcal{L}, \alpha \equiv_{z} \beta$ and $x \wedge y=z$ in $\mathcal{L}$ then there are $\gamma_{0}, \gamma_{1}, \gamma_{2}$ such that $\alpha \equiv_{x} \gamma_{0} \equiv_{y} \gamma_{1} \equiv_{x} \gamma_{2} \equiv_{y} \beta$ and $\Theta \cup\left\{\gamma_{0}, \gamma_{1}, \gamma_{2}\right\}$ is still an usl representation for $\mathcal{L}$. Moreover, these extensions can be found uniformly recursively in the given data.

Proof. This is a standard fact going back to Jonsson [1953] and can be found in Lerman [1983, Appendix B.2.5]. If $x \leq y$, there is nothing to be proved. Otherwise, the interpolants can be defined by letting $\gamma_{0}(w)$ be $\alpha(w)$ for $w \leq x$ and new values for $w \not \leq x$; $\gamma_{1}(w)=\gamma_{0}(w)$ for $w \leq y$ and new values otherwise; and $\gamma_{2}(w)=\beta(w)$ for $w \leq y$, $\gamma_{2}(w)=\gamma_{1}(w)$ if $w \leq x$ but $w \not \leq y$ and new otherwise. 
Proposition 5.6. If $\hat{\mathcal{L}} \subseteq_{l s l} \mathcal{L}$, a finite lattice, and $\Theta$ is an usl representation for $\mathcal{L}$ with $\alpha_{0}, \alpha_{1}, \beta_{0}, \beta_{1} \in \Theta$ such that $\forall w \in \hat{\mathcal{L}}\left(\alpha_{0} \equiv_{w} \alpha_{1} \rightarrow \beta_{0} \equiv_{w} \beta_{1}\right)$, then there is an usl representation $\tilde{\Theta} \supseteq \Theta$ for $\mathcal{L}$ with $\gamma_{0}, \gamma_{1} \in \tilde{\Theta}$ and $\hat{\mathcal{L}}$ homomorphisms $f, g, h: \Theta \rightarrow \tilde{\Theta}$ such that $f: \alpha_{0}, \alpha_{1} \mapsto \beta_{0}, \gamma_{1}, g: \alpha_{0}, \alpha_{1} \mapsto \gamma_{0}, \gamma_{1}$ and $h: \alpha_{0}, \alpha_{1} \mapsto \gamma_{0}, \beta_{1}$. Moreover, these extensions can be found uniformly recursively in the given data.

Proof. For each $\alpha \in \Theta$ and $x \in \mathcal{L}$ we set $f(\alpha)(x)=\beta_{0}(x)$ if $\alpha \equiv_{\hat{x}} \alpha_{0}$ and otherwise we let it be a new number that depends only on $\alpha(\hat{x})$, e.g. $\alpha(\hat{x})^{*}$. Note that which case of the definition applies for $f(\alpha)(x)$ depends only on $\alpha(\hat{x})$ and it can be an "old" value (i.e. one of some $\beta \in \Theta$ ) only in the first case. Thus, for $\alpha, \beta \in \Theta$,

$$
\text { (a) } \alpha \equiv_{\hat{x}} \beta \Leftrightarrow f(\alpha) \equiv_{x} f(\beta) \text { and (b) } f(\alpha) \equiv_{x} \beta \Rightarrow \alpha \equiv_{\hat{x}} \alpha_{0} \Rightarrow f(\alpha) \equiv_{x} \beta_{0} \text {. }
$$

Let $\Theta_{1}=\Theta \cup f[\Theta]$. We claim that $\Theta_{1}$ is an usl representation for $\mathcal{L}$ and $f$ is an $\hat{\mathcal{L}}$ homomorphism from $\Theta$ into $\Theta_{1}$. That $f$ is an $\hat{\mathcal{L}}$-homomorphism is immediate from the first clause in (1) and the fact (Lemma 5.3) that $\hat{x}=x$ for $x \in \hat{\mathcal{L}}$. We next check that $\Theta_{1}$ satisfies the properties required of an usl representation. Of course, $f(\alpha)(0)=0$ by definition for every $\alpha$ and differentiation is automatic as it extends $\Theta$.

First, to check the order property for $\Theta_{1}$ we consider any $x \leq y$ in $\mathcal{L}$. As $\Theta$ is already an usl representation for $\mathcal{L}$, it suffices to consider two cases for the pair of elements of $\Theta_{1}$ which are given as congruent modulo $y$ and show that in these two cases they are also congruent modulo $x$. The two cases are that (a) both are in $f[\Theta]$ and that (b) one is in $f[\Theta]$ and the other in $\Theta$. Thus it suffices to consider any $\alpha, \beta \in \Theta$, assume that (a) $f(\alpha) \equiv_{y} f(\beta)$ or (b) $f(\alpha) \equiv_{y} \beta$ and prove that (a) $f(\alpha) \equiv_{x} f(\beta)$ and (b) $f(\alpha) \equiv_{x} \beta$, respectively. For (a), we have by (1) that $\alpha \equiv_{\hat{y}} \beta$ and so by the order property for $\Theta$, $\alpha \equiv_{\hat{x}} \beta$. Thus $f(\alpha) \equiv_{x} f(\beta)$ by definition as required. As for (b), (1) tells us here that $\alpha \equiv_{\hat{y}} \alpha_{0}$ and $\beta \equiv_{y} f(\alpha) \equiv_{y} \beta_{0}$ (and therefore $\beta \equiv_{x} \beta_{0}$ ). Now by Lemma $5.3 \alpha \equiv_{\hat{x}} \alpha_{0}$ so $f(\alpha) \equiv_{x} \beta_{0}$ and so $f(\alpha) \equiv_{x} \beta$ as required.

Next we verify the join property for $x \vee y=z$ in $\mathcal{L}$ and two elements of $\Theta_{1}$ (not both in $\Theta$ ) in the same two cases. For (a) we have that $f(\alpha) \equiv_{x, y} f(\beta)$ and so as above $\alpha \equiv_{\hat{x}, \hat{y}} \beta$. Now by the join property in $\Theta$ and Lemma $5.3, \alpha \equiv_{\hat{z}} \beta$ and so $f(\alpha) \equiv_{z} f(\beta)$ as required. For (b) using (1b) and Lemma 5.3 again we have that $f(\alpha) \equiv_{x, y} \beta \Rightarrow$ $\alpha \equiv_{\hat{x}, \hat{y}} \alpha_{0} \Rightarrow \alpha \equiv_{\hat{z}} \alpha_{0} \Rightarrow f(\alpha) \equiv_{z} \beta_{0}$ while it also tells us that $\beta \equiv_{x, y} f(\alpha) \equiv_{x, y} \beta_{0}$ as required. Note that clearly $f\left(\alpha_{0}\right)=\beta_{0}$. We let $\gamma_{1}=f\left(\alpha_{1}\right)$ and so have the first function and (partial) extension of $\Theta$ required in the Proposition.

We now define $h$ on $\Theta_{1}$ as we did $f$ on $\Theta$ using $\alpha_{1}$ and $\beta_{1}$ in place of $\alpha_{0}$ and $\beta_{0}$, respectively: $h(\alpha)(x)=\beta_{1}(x)$ if $\alpha \equiv_{\hat{x}} \alpha_{1}$ and otherwise we let it be a new number that depends only on $\alpha(\hat{x})$, e.g. $\alpha(\hat{x})^{* *}$. Let $\Theta_{2}=\Theta_{1} \cup h\left[\Theta_{1}\right]$. As above, $\Theta_{2}$ is an usl representation for $\mathcal{L}$ and $h$ is an $\hat{\mathcal{L}}$-homomorphism from $\Theta_{1}$ (and so $\Theta$ ) into $\Theta_{2}$ taking $\alpha_{1}$ to $\beta_{1}$. We let $\gamma_{0}=h\left(\alpha_{0}\right)$ and so have the third function and (partial) extension of $\Theta$ required in the Proposition. As above in (1), we have for any $\alpha, \beta \in \Theta_{1}$ and $x \in \mathcal{L}$,

$$
\text { (a) } \alpha \equiv_{\hat{x}} \beta \Leftrightarrow h(\alpha) \equiv_{x} h(\beta) \text { and (b) } h(\alpha) \equiv_{x} \beta \Rightarrow \alpha \equiv_{\hat{x}} \alpha_{1} \Rightarrow h(\alpha) \equiv_{x} \beta_{1} \text {. }
$$


Applying the second clause to $\gamma_{0}=h\left(\alpha_{0}\right)$ and first to any $\beta \in \Theta_{1}$ and then, in particular to $\gamma_{1}$ we have

$$
\text { (a) } \gamma_{0} \equiv_{x} \beta \Rightarrow \alpha_{0} \equiv_{\hat{x}} \alpha_{1} \Rightarrow f\left(\alpha_{1}\right)=\gamma_{1} \equiv_{x} \beta_{0} \text { and (b) } \gamma_{0} \equiv_{x} \gamma_{1} \Leftrightarrow \alpha_{0} \equiv_{\hat{x}} \alpha_{1} \text {. }
$$

To see the right to left direction of the second clause, note that $\alpha_{0} \equiv_{\hat{x}} \alpha_{1}$ implies that $\gamma_{0} \equiv_{x} \beta_{1}$ and $\gamma_{1} \equiv_{x} \beta_{0}$ by the definitions of $h$ and $f$, respectively, while it also implies that $\beta_{0} \equiv_{\hat{x}} \beta_{1}$ by the basic assumption of the Proposition. Thus, as $\Theta$ is an usl representation of $\mathcal{L}$ and $x \leq \hat{x}, \beta_{0} \equiv_{x} \beta_{1}$ and $\gamma_{0} \equiv_{x} \gamma_{1}$.

Finally, we define $g$ on $\alpha \in \Theta_{2}$ by setting $g(\alpha)(x)=\gamma_{0}(x)$ if $\alpha \equiv_{\hat{x}} \alpha_{0}$. If $\alpha \neq_{\hat{x}} \alpha_{0}$ but $\alpha \equiv_{\hat{x}} \alpha_{1}$ then $g(\alpha)(x)=\gamma_{1}(x)$. Otherwise, we let $g(\alpha)(x)$ be a new number that depends only on $\alpha(\hat{x})$, e.g. $\alpha(\hat{x})^{* * *}$. Note that if $\alpha \equiv_{\hat{x}} \alpha_{1}$ then we always have $g(\alpha) \equiv_{x} \gamma_{1}$ as if $\alpha \equiv_{\hat{x}} \alpha_{0}$ as well then, by (3b), $\gamma_{0} \equiv_{x} \gamma_{1}$. Thus $g\left(\alpha_{0}\right)=\gamma_{0}$ and $g\left(\alpha_{1}\right)=\gamma_{1}$ as required. It is also obvious that $g$ is an $\hat{\mathcal{L}}$-homomorphism of $\Theta_{2}$ (and so $\Theta$ ) into $\Theta_{3}=\Theta_{2} \cup g\left[\Theta_{2}\right]$ as by definition and Lemma 5.3, $\alpha \equiv_{\hat{x}} \beta \Rightarrow g(\alpha) \equiv_{\hat{x}} g(\beta)$ for any $x \in \mathcal{L}$. Indeed, for any $\alpha, \beta \in \Theta_{2}$ and $x \in \mathcal{L}$

$$
\alpha \equiv_{\hat{x}} \beta \Leftrightarrow g(\alpha) \equiv_{x} g(\beta) .
$$

To see the right to left direction here, note that if either of $g(\alpha)$ or $g(\beta)$ is new for $g$ at $x$ (i.e. of the form $\left.\delta(\hat{y})^{* * *}\right)$ then clearly both are. In this case, $\alpha \equiv_{\hat{x}} \beta$ by definition. Otherwise, either they are both congruent to $\alpha_{0}$ or both to $\alpha_{1}$ and so congruent to each other mod $\hat{x}$. The point here is that if one is congruent to $\alpha_{0}$ and the other to $\alpha_{1}$ but not $\alpha_{0}$ at $\hat{x}$ then by definition $\gamma_{0} \equiv_{x} \gamma_{1}$ and so by (3b), $\alpha_{0} \equiv_{\hat{x}} \alpha_{1}$ for a contradiction.

Thus we only need to verify that $\Theta_{3}$ is an usl representation of $\mathcal{L}$. We consider any $\alpha, \beta \in \Theta_{2}$ and divide the verifications into cases (a) and (b) as before with the former considering $g(\alpha)$ and $g(\beta)$ and the latter $g(\alpha)$ and $\beta$. These cases may then be further subdivided.

We begin with the order property and so $x \leq y$ in $\mathcal{L}$.

(a) If $g(\alpha) \equiv_{y} g(\beta)$ then, by (4), $\alpha \equiv_{\hat{y}} \beta$ and so $\alpha \equiv_{\hat{x}} \beta$ as $\hat{x} \leq \hat{y}$ (Lemma 5.3) and $\Theta_{2}$ is an usl representation of $\mathcal{L}$. Thus, again by (4) $g(\alpha) \equiv_{x} g(\beta)$ as required.

(b) If $g(\alpha) \equiv_{y} \beta$ then by definition they are congruent modulo $y$ to $\gamma_{i}$ (for some $i \in\{0,1\})$ and $\alpha$ is congruent to $\alpha_{i}$ at $\hat{y}$. Thus $\alpha \equiv_{\hat{x}} \alpha_{i}$ as $\hat{x} \leq \hat{y}$ and $\Theta_{2}$ is an usl representation so $g(\alpha) \equiv_{x} \gamma_{i}$ by definition. Similarly, as $x \leq y, \beta \equiv_{x} \gamma_{i}$ as well.

Now for the join property for $x \vee y=z$ in $\mathcal{L}$.

(a) If $g(\alpha) \equiv_{x, y} g(\beta)$ then, as above, $\alpha \equiv_{\hat{x}, \hat{y}} \beta$. As $\hat{x} \vee \hat{y}=\hat{z}$ by Lemma 5.3 and $\Theta_{2}$ is an usl representation, $\alpha \equiv_{\hat{z}} \beta$ and so by $(4) g(\alpha) \equiv_{z} g(\beta)$ as required.

(b) If $g(\alpha) \equiv_{x, y} \beta$ then again $\alpha \equiv_{\hat{x}} \alpha_{i}$ and $\alpha \equiv_{\hat{y}} \alpha_{j}$ for some $i, j \in\{0,1\}$ and $g(\alpha) \equiv_{x} \beta \equiv_{x} \gamma_{i}$ while $g(\alpha) \equiv_{y} \beta \equiv_{y} \gamma_{j}$. If $i=j$ then $\alpha \equiv_{\hat{x}, \hat{y}} \alpha_{i}$ and so $\alpha \equiv_{\hat{z}} \alpha_{i}$ and $g(\alpha) \equiv_{z} \gamma_{i} \equiv_{z} \beta$ as required.

On the other hand, suppose (without loss of generality) that $\alpha \equiv_{\hat{x}} \alpha_{0}$ and so $\beta \equiv_{x}$ $g(\alpha) \equiv_{\hat{x}, x} \gamma_{0}=h\left(\alpha_{0}\right)$ while $\alpha_{0} \not_{\hat{y}} \alpha \equiv_{\hat{y}} \alpha_{1}$ and so $\beta \equiv_{y} g(\alpha) \equiv_{\hat{y}, y} \gamma_{1}=f\left(\alpha_{1}\right)$. If $\beta \in \Theta_{1}$ then by (4a) $\alpha_{0} \equiv_{\hat{x}} \alpha_{1}$ and so $\alpha \equiv_{\hat{x}} \alpha_{1}$. As our assumption is that $\alpha \equiv_{\hat{y}} \alpha_{1}$ we have (by 
the join property in $\left.\Theta_{2}\right)$ that $\alpha \equiv_{\hat{z}} \alpha_{1}$ and so $g(\alpha) \equiv_{z} \gamma_{1}$. As $\alpha_{0} \equiv_{\hat{x}} \alpha_{1}(3 \mathrm{~b})$ tells us that $\gamma_{0} \equiv_{x} \gamma_{1}$. Our assumptions then say that $\beta \equiv_{x, y} \gamma_{1}$ and so $\beta \equiv_{z} \gamma_{1}$ as required. Thus we may assume that $\beta=h(\delta)$ for some $\delta \in \Theta_{1}$.

We now have $h(\delta)=\beta \equiv_{x} g(\alpha) \equiv_{x} \gamma_{0}=h\left(\alpha_{0}\right) \in \Theta_{1}$ and so by (2a) applied to $h(\delta) \equiv_{x} h\left(\alpha_{0}\right)$ with $\delta$ for $\alpha$ and $\alpha_{0}$ for $\beta$ we see that $\delta \equiv_{\hat{x}} \alpha_{0}$. We also have $h(\delta)=\beta \equiv_{y}$ $g(\alpha) \equiv_{\hat{y}, y} \gamma_{1}=f\left(\alpha_{1}\right)$. Applying (2b) to $h(\delta) \equiv_{y} \gamma_{1}$ with $\delta$ for $\alpha$ and $\gamma_{1} \in \Theta_{1}$ for $\beta$, we see that $\delta \equiv_{\hat{y}} \alpha_{1}$ and $h(\delta) \equiv_{y} \beta_{1}$ and so $\beta_{1} \equiv_{y} \gamma_{1}=f\left(\alpha_{1}\right)$. Now applying (1b) with $\alpha_{1}$ for $\alpha$ and $\beta_{1} \in \Theta$ for $\beta$, we have that $\alpha_{1} \equiv_{\hat{y}} \alpha_{0}$. As this contradicts our (without loss of generality) assumption, we are done.

We complete our lattice theoretic material by supplying a proof of Lemma 1.4.

Lemma 1.4: The class of finite lattices (with 0 and 1) has the amalgamation property, i.e. if $\mathcal{A}, \mathcal{B}_{0}$ and $\mathcal{B}_{1}$ are finite lattices and $f_{0}, f_{1}$ are embeddings of $\mathcal{A}$ into $\mathcal{B}_{0}$ and $\mathcal{B}_{1}$, respectively, then there is a finite lattice $\mathcal{C}$ and embeddings $g_{0}$ and $g_{1}$ of $\mathcal{B}_{0}$ and $\mathcal{B}_{1}$, respectively, into $\mathcal{C}$ such that $g_{0} f_{0}\left\lceil\mathcal{A}=g_{1} f_{1}\lceil\mathcal{A}\right.$.

Proof. To simplify the notation we assume without loss of generality that the embeddings $f_{0}$ and $f_{1}$ are the inclusion maps and that the elements of $\mathcal{A}$ are the only ones that $\mathcal{B}_{0}$ and $\mathcal{B}_{1}$ have in common. We begin with a partial lattice $\mathcal{P}$ whose universe is the union of those of $\mathcal{B}_{0}$ and $\mathcal{B}_{1}$. We define an order on $\mathcal{P}$ that coincides with the one on $\mathcal{B}_{i}$ for $x, y$ both in one $\mathcal{B}_{i}$ and otherwise (say $x \in \mathcal{B}_{i}-\mathcal{A}$ and $y \in \mathcal{B}_{1-i}-\mathcal{A}$ ) we set $x<y \Leftrightarrow \exists a \in \mathcal{A}\left(x<_{\mathcal{B}_{i}} a \& a<_{\mathcal{B}_{1-i}} y\right)$. This relation is clearly transitive and we claim it preserves both join and meet from each $\mathcal{B}_{i}$. First, if $x, y \in \mathcal{B}_{i}$ and $x \wedge y=z$ in $\mathcal{B}_{i}$ then $z$ is also the greatest lower bound of $x$ and $y$ in $\mathcal{P}$. Clearly $z \leq x, y$. So suppose $w \leq x, y$ is in $\mathcal{P}$. If $w \in \mathcal{B}_{i}$ then, of course, $w \leq z$. If $w \in \mathcal{P}-\mathcal{B}_{i}$ then there are $a_{x}, a_{y} \in \mathcal{A}$ such that $w<a_{x} \leq x$ and $w<a_{y} \leq y$ so $w \leq a_{x} \wedge_{\mathcal{A}} a_{y} \leq a_{x} \wedge_{\mathcal{B}_{i}} a_{y} \leq x \wedge_{\mathcal{B}_{i}} y=z$. The argument for preserving join is similar.

Now let $\mathcal{C}$ be the set of ideals of $\mathcal{P}$, i.e. the subsets of $\mathcal{P}$ closed downward and under join when defined in $\mathcal{P}$. We first note that $\mathcal{C}$ is clearly a lattice with order given by containment and the operations on $X, Y \in \mathcal{C}$ given by $X \wedge Y=X \cap Y$ and $X \vee Y$ equals the ideal in $\mathcal{P}$ generated by $X \cup Y$ (i.e. we close downward and under join when defined).

Finally, we define the required maps $g_{i}: \mathcal{B}_{i} \rightarrow \mathcal{C}$ as the restrictions (to $\mathcal{B}_{i}$ ) of a single one-one $g: \mathcal{P} \rightarrow \mathcal{C}$ defined by sending $p \in \mathcal{P}$ to $\left\{q \in \mathcal{P} \mid q \leq_{\mathcal{P}} p\right\}$ (the ideal generated by $p$ ). We show that $g$ preserves join and meet in $\mathcal{P}$ when they exist and so its restrictions to $\mathcal{B}_{i}$ are lattice embeddings. If $p \wedge q=r$ in $\mathcal{P}$ then it is clear that $g(p) \cap g(q)=g(r)$ by the definition of meet in $\mathcal{P}$ as required. As for join, if $p \vee q=r$ in $\mathcal{P}$ then $g(r)$ is an ideal of $\mathcal{P}$ that contains both $g(p)$ and $g(q)$. On the other hand, any ideal of $\mathcal{P}$ containing both $p$ and $q$ must contain $r$ by the definition of ideals in $\mathcal{P}$. Thus $g(r)=g(p) \vee_{\mathcal{C}} g(q)$ as required. 


\section{Usls and other questions}

There are now two obvious questions about the possible countable initial segments of the hyperdegrees. The first asks about lattice initial segments.

Question 6.1. What are the lattice initial segments of $\mathcal{D}_{h}$ ? In particular, are there any which are not sublattices of some hyperarithmetic lattice?

We do not even have any candidates for additional lattices isomorphic to initial segments of $\mathcal{D}_{h}$.

The second natural line of inquiry asks about usl initial segments. In $\mathcal{D}_{T}$, there is no difference in the results: Every countable usl is isomorphic to an initial segment of $\mathcal{D}_{T}$. Of course, we have seen (Theorem 1.1) that not every countable lattice is isomorphic to an initial segment of $\mathcal{D}_{h}$. Given our Theorem 1.2, however, the conjecture might be that every subuppersemilattice (susl) of a hyperarithmetic lattice is isomorphic to an initial segment of $\mathcal{D}_{h}$. Now our proof actually makes significant use of the existence of infima in $\mathcal{L}$ at various points. As it turns out, the assumption is essential, at least at this level of generality even if we require $\mathcal{L}$ to be locally finite as well.

Proposition 6.2. There is a susl $\mathcal{L}^{\prime}$ of a locally finite recursive lattice $\mathcal{L}$ which is not isomorphic to any initial segment of $\mathcal{D}_{h}$.

Proof. The construction is an elaboration of that referred to in Theorem 1.1 that exploits the possibility of exact pairs for ideals in usls to make the initial lattice locally finite. The basic construction of the finitely generated successor model of Shore [1981] as modified in Shore [2007], [2008] begins with special elements designated by $d_{0}, e_{0}, e_{1}, f_{0}, f_{1}, p$ and $q$. They contain a sequence $d_{n}$ of elements of order type $\omega$ generated by the special elements as follows:

$(*)\left(d_{2 n} \vee e_{0}\right) \wedge f_{1}=d_{2 n+1}$ and

$(* *)\left(d_{2 n+1} \vee e_{1}\right) \wedge f_{0}=d_{2 n+2}$.

In addition we require that $p \nsupseteq q$ and $p \vee d_{n} \geq q$ for each $n$. We then code a set $X$ by adding two additional special elements $c_{X}$ and $\bar{c}_{X}$ such that $d_{n} \leq c_{X}$ for $n \in X$, $d_{n} \wedge c_{X}=0$ for $n \notin X, d_{n} \leq \bar{c}_{X}$ for $n \notin X$ and $d_{n} \wedge \bar{c}_{X}=0$ for $n \in X$. (So, in particular, $\exists x\left(0<x \leq d_{n}, c_{X}\right) \rightarrow d_{n} \leq c_{X}$ and $\left.\exists x\left(0<x \leq d_{n}, \bar{c}_{X}\right) \rightarrow d_{n} \leq \bar{c}_{X}.\right)$

We adjust this procedure to make the lattice locally finite. In place of $d_{0}$ we have a set of elements $d_{i, 0}$ for $i \in \omega$. For each $i$ the sequence generated by the schemes $(*)$ and $(* *)$ now terminates after $i$ steps producing sequences $d_{i, 0}, \ldots, d_{i, i}$ of length $i+1$ by having $\left(d_{i, i} \vee e_{0}\right) \wedge f_{1}=0$ if $i$ is even and $\left(d_{i, i} \vee e_{1}\right) \wedge f_{0}=0$ if $i$ is odd. We now require that $p \vee d_{i, j} \geq q$ for every $j \leq i \in \omega$ and add on new elements $\hat{p} \ngtr \hat{q}$ such that $\hat{p} \vee d_{i, j} \geq \hat{q}$ if and only if $j=i$. In place of $c_{X}$ and $\bar{c}_{X}$ we have one fixed pair $c$ and $\bar{c}$ that 
are above all the $d_{i, 0}$ and no other of the previously mentioned elements. We complete this description to a lattice $\mathcal{L}$ in a way that respects the given ordering and specified join and meet relations and makes $c \wedge \bar{c}$ a minimal upper bound of the ideal generated by the $d_{i, 0}$ with no other nonzero elements below it. We give more details after we see what properties are needed to make our coding of sets in a susl of $\mathcal{L}$ be sufficiently flexible to show that some such are not isomorphic to initial segments of $\mathcal{D}_{h}$.

Given a set $X$ we want to code $X$ into a susl $\mathcal{K}$ of $\mathcal{L}$ by taking the susl of $\mathcal{L}$ generated by the special elements $c, \bar{c}, e_{0}, e_{1}, f_{0}, f_{1}, p, q, \hat{p}$ and $\hat{q}$ and the $d_{i, j}$ for $i \in X$. In particular, $c$ and $\bar{c}$ will now be an exact pair for the ideal generated by the $d_{i, 0}$ for $i \in X$. We want to guarantee that $n \in X \Leftrightarrow$ there is a sequence $x_{0}, \ldots, x_{n}$ with $x_{0} \leq c, \bar{c} ; x_{2 m+1} \leq x_{2 m} \vee e_{0}, f_{1}$ and $x_{2 m+2} \leq x_{2 m+1} \vee e_{1}, f_{0}$ for $2 m+1,2 m+2 \leq n ; x_{m} \vee p \geq q$ for $m \leq n$ and $x_{n} \vee \hat{p} \geq \hat{q}$.

We claim that in this case $X$ is $\Pi_{1}^{1}$ in the top $G$ of any embedding of $\mathcal{K}$ as an initial segment. As existential quantification over sets hyperarithmetic in $G$ and the relation $A \leq_{h} B$ for sets given as hyperarithmetic in $G$ are both $\Pi_{1}^{1}$ in $G$ and the join operator is recursive (on indices), it is clear that the specified relation on $n$ is $\Pi_{1}^{1}(G)$ and that it holds of every $n \in X$. What remains to verify is that it holds only of $n \in X$. So suppose there are $x_{0}, \ldots, x_{n}$ as described. For $x_{0} \leq c, \bar{c}$, we want $x_{0}$ to be the join of finitely many $d_{i, 0}$ by making these the only elements of $\mathcal{K}$ below both $c$ and $\bar{c}$. Say for definiteness that $x_{0}=d_{i_{1}, 0} \vee \cdots \vee d_{i_{k}, 0}$. We also arrange our lattice so that $\left(x_{0} \vee e_{0}\right) \wedge f_{1}=d_{i_{1}, 1} \vee \cdots \vee d_{i_{k}, 1}$ and so $x_{1} \leq d_{i_{1}, 1} \vee \cdots \vee d_{i_{k}, 1}$. In general, we arrange our lattice so that $\left(x_{2 n} \vee e_{0}\right) \wedge f_{1}=$ $d_{i_{1}, 2 n+1} \vee \cdots \vee d_{i_{k}, 2 n+1}$ and $\left(x_{2 n+1} \vee e_{1}\right) \wedge f_{0}=d_{i_{1}, 2 n+2} \vee \cdots \vee d_{i_{k}, 2 n+2}$ where we understand that for $m>i, d_{i, m}=0$. Thus $x_{m} \leq d_{i_{1}, m} \vee \cdots \vee d_{i_{k}, m}$. The requirements that $x_{m} \vee p \geq q$ guarantee that $x_{m}>0$ and, by making the $d_{i, j}$ minimal elements of the lattice, they must be above some nonzero $d_{i_{k}, m}$. Finally, we guarantee that the only way such an $x_{m}$ can join $\hat{p}$ above $\hat{q}$ is for it to be above some $d_{i_{k}, i_{k}}$ but these elements are in $\mathcal{K}$ if and only if $i_{k} \in X$ as required.

Now to be more specific about the structure of $\mathcal{L}$ we specify its elements and the order on them that will give a lattice with all the desired properties. We begin, of course, with 0 and 1 . The elements $e_{0}, e_{1}, p, q, \hat{p}, \hat{q}$ and $d_{i, j}$ (for $j \leq i \in \omega$ ) are minimal nonzero elements of $\mathcal{L}$. We extend the $d_{i, j}$ freely to an usl $\mathcal{L}^{\prime}$ by taking all formal finite joins. This imposes a lattice structure on this set as well since each of the new elements has only finitely many elements below it. We next let $\hat{p}$ act on the usl $\mathcal{L}^{\prime}$ as an order isomorphism under join (so for $x, y \in \mathcal{L}^{\prime}, x \vee \hat{p} \leq y \vee \hat{p} \Leftrightarrow x \leq y$ ). Let $\mathcal{L}^{\prime \prime}$ be the susl of $\mathcal{L}^{\prime}$ generated by the $d_{i, j}$ with $j<i$. For $x \in \mathcal{L}^{\prime \prime}$, no elements other than $\hat{p}$ and those $y \leq x$ are below $x \vee \hat{p}$. For $x \in \mathcal{L}^{\prime}-\mathcal{L}^{\prime \prime}$ we also put $x \vee \hat{p} \geq \hat{q}$. Joining $e_{0}$ with members of $\mathcal{L}^{\prime}$ also acts as an order isomorphism except that, for any $m \in \omega$ and $\left\langle i_{k}\right\rangle,\left\langle j_{k}\right\rangle \in \omega^{m}$ such that $\forall k<m\left(j_{k} \leq i_{k}\right)$, we make the following identification:

$e_{0} \vee \bigvee\left\{d_{i_{k}, j_{k}} \mid k<m\right\}=e_{0} \vee \bigvee\left\{d_{i_{k}, j_{k}} \mid k<m\right\} \vee \bigvee\left\{d_{i_{k}, j_{k}+1} \mid k<m, j_{k}<i_{k}\right.$ and $j_{k}$ is even $\}$

We do the same for joining with $e_{1}$ except that we change "even" to "odd". We let $f_{0}\left(f_{1}\right)$ be above the usl generated by the $d_{i, j}$ for odd (even) $j \leq i$ and put in a new 
element $c \wedge \bar{c}$ (below both $c$ and $\bar{c}$ ) which is above the usl generated by the $d_{i, 0}$. Any order relation $x \leq y$ not dictated by these definitions is taken to be false. So for example, $e_{0} \vee e_{1}=1=f_{0} \vee f_{1}, e_{0} \wedge e_{1}=0=f_{0} \wedge f_{1}, x \vee p=1$ for any $x \neq 0, d_{i, i} \vee \hat{p}=1$ for every $i$, etc. It is tedious but straightforward to verify that the partial order so defined imposes on the elements described a lattice structure $\mathcal{L}$ (i.e. for every $x, y \in \mathcal{L}$ there is a least upper bound and a greatest lower bound for the pair in the defined ordering) that has all the desired properties.

If we now take $X$ to be, for example, the complement of $\mathcal{O}$, then the top of any embedding of $\mathcal{K}$ as an initial segment would be above $\mathcal{O}$ and so the degrees below it could not be isomorphic to $\mathcal{K}$ (as, for example, every countable partial order can be embedded in $\mathcal{D}_{h}$ below $\operatorname{deg}_{h} \mathcal{O}$ by Feferman [1965]).

On the other hand, there are initial segments of $\mathcal{D}_{h}$ which are not lattices. Indeed, the usual proof that $\mathcal{D}_{T}$ is not a lattice can be carried out for $\mathcal{D}_{h}$ by using Cohen forcing in the hyperarithmetic setting to show that the degrees below $\mathcal{O}$ are not a lattice (as is pointed out in Odifreddi [1983a, Proposition 8.3(b)]. Thus we have our next question.

Question 6.3. Which countable usls are isomorphic to initial segments of $\mathcal{D}_{h}$ ?

This question seems wide open and, by the above Proposition, must need some new construction technique. One attractive possibility would be a positive answer to the following.

Question 6.4. Is every hyperarithmetic usl isomorphic to an initial segment of $\mathcal{D}_{h}$ ?

Here even the simplest example seems to need some new idea.

Question 6.5. Is the usl consisting precisely of an initial segment $x_{n}$ of type $\omega$ with an exact pair $x, y$ and their join (i.e. $0=x_{0}, \forall n\left(x_{n}<x_{n+1} \leq x, y\right)$ and $x \vee y=1$ ) isomorphic to an initial segment of $\mathcal{D}_{h}$ ?

On the other hand, we have no particularly plausible candidate for a counterexample to Question 6.4.

\section{References}

[1] Abraham, U. and Shore, R. A. [1986], Initial segments of the degrees of size $\aleph_{1}$, Israel J. Math. 53, 1-51.

[2] Abraham, U. and Shore, R. A. [1986a], The degrees of constructibility below a Cohen real, J. Lon. Math. Soc. (3) 53, 193-208.

[3] Adamowicz, Z. [1976], On finite lattices of degrees of constructibility, J. Symb. Logic 41, 313-322. 
[4] Adamowicz, Z. [1977], Constructible semi-lattices of degrees of constructibility, in Set Theory and Hierarchy Theory V, Lachlan, Srebny and Zarach eds., LNM 619, Springer-Verlag, Berlin.

[5] Balcar, B. and Hajek, P. [1978], On sequences of degrees of constructibility, Z. Math. Logik Grundlag. Math. 24, 291-296.

[6] Cohen, P. [1966], Set Theory and the Continuum Hypothesis, Benjamin, New York.

[7] Dorais, F. [2007], Souslin Trees and Degrees of Constructibility, Ph. D. Thesis, Dartmouth College.

[8] Farrington, P. [1983], Hinges and automorphisms of the degrees of constructibility, J. Lon. Math. Soc. (2) 28, 193-202.

[9] Farrington, P. [1984], First order theory of the c-degrees, Z. Math. Logik Grundlag. Math. 30, 437-446.

[10] Feferman, S. [1965], Some applications of the notion of forcing and generic sets, Fund. Math. 56, 325-45.

[11] Gandy, R. O. and Sacks, G. E. [1967], A minimal hyperdegree, Fund. Math. 61, 215-223.

[12] Grätzer, G. [2003], General Lattice Theory, 2nd ed., Birkhäuser Verlag, Basel.

[13] Groszek, M. S. and Shore, R. A. [1988], Initial segments of the degrees of constructibility, Israel J. Math. 63, 149-177.

[14] Groszek, M. S. and Slaman, T. A. [1983], Independence results on the global structure of the Turing degrees, Trans. Am. Math. Soc. 277, 579-588.

[15] Hodges, W. [1993], Model Theory, Encyclopedia of Mathematics and its Applications 42, Cambridge University Press, Cambridge, England.

[16] Jónsson, B. [1953], On the representations of lattices, Math. Scand. 1, 193-206.

[17] Kjos-Hanssen, B. [2002], Lattice initial segments of the Turing degrees, Ph. D. Thesis, University of California, Berkeley.

[18] Kjos-Hanssen, B. [2003], Local initial segments of the Turing degrees, B. Symb. Logic 9, 26-36.

[19] Kleene, S. C. and Post, E. L. [1954], The upper semi-lattice of degrees of recursive unsolvability, Ann. Math. (2) 59, 379-407.

[20] Lachlan, A. H. [1968], Distributive initial segments of the degrees of unsolvability, Z. Math. Logik Grund. Math. 14, 457-472. 
[21] Lachlan, A. H. and Lebeuf, R. [1976], Countable initial segments of the degrees of unsolvability, J. Symb. Logic 41, 289-300.

[22] Lerman, M. [1971], Initial segments of the degrees of unsolvability, Ann. Math. 93, 365-89.

[23] Lerman, M. [1983], Degrees of Unsolvability, Perspectives in Mathematical Logic, Springer-Verlag, Berlin.

[24] Lubarsky, R. [1987], Lattices of c-degrees, Ann. Pure and Applied Logic 36, 115-118.

[25] Miller, R. G., Nies, A. O. and Shore, R. A. [2004], The $\forall \exists$-theory of $\mathcal{R}(\leq, \vee, \wedge)$ is undecidable, Trans. Am. Math. Soc. 356, 3025-3067.

[26] Nerode, A. and Shore, R. A. [1980], Second order logic and first order theories of reducibility orderings, in The Kleene Symposium, J. Barwise, H. J. Keisler and K. Kunen, eds., North-Holland, Amsterdam, 181-200.

[27] Odifreddi, P. [1983], Forcing and reducibilities, J. Symb. Logic 48, 288-310.

[28] Odifreddi, P. [1983a], Forcing and reducibilities II: forcing in fragments of analysis, J. Symb. Logic 48, 724-743.

[29] Sacks, G. E. [1963], Degrees of unsolvability, Annals of Math. Studies 55, Princeton Univ. Press, Princeton, New Jersey.

[30] Sacks, G. E. [1971], Forcing with perfect closed sets, in Axiomatic set Theory, Proc. Symp. Pure Math. XII, Part 1, AMS, Providence, Rhode Island.

[31] Sacks, G. E. [1972], review of Thomason [1970], Math. Reviews, issue 2, MR0288027(44\#5225).

[32] Sacks, G. E. [1990], Higher Recursion Theory, Perspectives in Mathematical Logic, Springer-Verlag, Berlin.

[33] Selivanov, V. L. [1988], Algorithmic complexity of algebraic systems, Mat. Zametki 44, 823-832, 863; translation in Math. Notes 44, 944-950 (1989)

[34] Shore, R. A. [1978], On the $\forall \exists$-sentences of $\alpha$-recursion theory, in Generalized Recursion Theory II, J. E. Fenstad, R. O. Gandy and G. E. Sacks eds., Studies in Logic and the Foundations of Mathematics 94, North-Holland, Amsterdam, 331-354.

[35] Shore, R. A. [1981], The theory of the degrees below 0', J. Lon. Math. Soc. (3) 24, $1-14$.

[36] Shore, R. A. [2007], Local definitions in degree structures: the Turing jump, hyperdegrees and beyond, B. Symb. Logic 13, 226-239. 
[37] Shore, R. A. [2008], Rigidity and biinterpretability in the hyperdegrees, to appear.

[38] Simpson, M. F. [1985], Arithmetic Degrees: Initial Segments, $\omega$-REA Operators and the $\omega$-jump, Ph. D. thesis, Cornell University.

[39] Spector, C. [1956], On degrees of recursive unsolvability, Ann. Math. (2) 64, 581-592.

[40] Thomason, S. K. [1967], The forcing method and the upper semilattice of hyperdegrees, Trans. Am. Math. Soc. 129, 38-57.

[41] Thomason, S. K. [1969], A note on non-distributive sublattices of degrees and hyperdegrees, Canad. J. Math. 21, 147-148.

[42] Thomason, S. K. [1970], On initial segments of hyperdegrees, J. Symb. Logic 35, 189-197. 